\title{
Static and Vibrational Analysis of Partially Composite Beams Using the Weak-Form Quadrature Element Method
}

\author{
Zhiqiang Shen and Hongzhi Zhong \\ Department of Civil Engineering, Tsinghua University, Beijing 100084, China \\ Correspondence should be addressed to Hongzhi Zhong,hzz@mail.tsinghua.edu.cn
}

Received 29 August 2011; Revised 7 November 2011; Accepted 21 November 2011

Academic Editor: Trung Nguyen Thoi

Copyright (C) 2012 Z. Shen and H. Zhong. This is an open access article distributed under the Creative Commons Attribution License, which permits unrestricted use, distribution, and reproduction in any medium, provided the original work is properly cited.

\begin{abstract}
Deformation of partially composite beams under distributed loading and free vibrations of partially composite beams under various boundary conditions are examined in this paper. The weak-form quadrature element method, which is characterized by direct evaluation of the integrals involved in the variational description of a problem, is used. One quadrature element is normally sufficient for a partially composite beam regardless of the magnitude of the shear connection stiffness. The number of integration points in a quadrature element is adjustable in accordance with convergence requirement. Results are compared with those of various finite element formulations. It is shown that the weak form quadrature element solution for partially composite beams is free of slip locking, and high computational accuracy is achieved with smaller number of degrees of freedom. Besides, it is found that longitudinal inertia of motion cannot be simply neglected in assessment of dynamic behavior of partially composite beams.
\end{abstract}

\section{Introduction}

Composite structures of different materials with interlayer slip have found a wide range of applications in structural engineering, such as in building, bridge and shelter construction. Common examples of such structures in civil engineering are steel-concrete composite beams (or decks) in buildings and bridges, wood-concrete floor or wall systems, and so forth. Shear connectors are usually used to connect different portions of these composite structures such as headed studs for steel-concrete composite beams. Since the shear connector is not completely rigid in practice, an interlayer slip, also known as partial interaction, often develops. In some cases, the interlayer slip significantly affects the mechanical behavior of composite systems. Thus, the structural model must account for the interlayer slip between the components, and this is very important in analysis and design of such partially composite structural systems. 
The problem of partially composite structures has been studied for several decades. One of the first analytical theories for partial interaction of composite beams was developed by Newmark et al. [1] who focussed their attention on steel-concrete composite beams with flexible shear connection based on Euler-Bernoulli beam hypothesis. Newmark's model was later extended by many researchers. Recently, analytical solutions for static and dynamic responses of partially composite beams (PCBs) were developed by Girhammar et al. $[2,3]$. Analytical solutions for static and dynamic responses of partially composite beams based on Timoshenko beam theory were obtained by $\mathrm{Xu}$ and $\mathrm{Wu}$ [4]. However, these analytical solutions are restricted to beams with single span and simple loads. Nonlinear analysis and linear elastic analysis of the engineering problems under more general circumstances have to resort to numerical tools [5-18] among which the finite element method (FEM) appears to be widely used [5-7]. It is well known that low-order finite element models of composite beams experience the so-called "slip locking" phenomenon for large values of the shear connection stiffness [8-10]. Possible strategies in finite element analysis to reduce or even to eliminate slip locking are lowering the degree of interpolation functions for the slip or introducing elements with larger numbers of degrees of freedom. These strategies proceed unexceptionally at the expense of increasing the total degrees of freedom of the finite element model to achieve an acceptable accuracy. Furthermore, the demand of more degrees of freedom is exacerbated when nonlinear analysis of partially composite beams is performed.

Another issue that may be worth mentioning is the evaluation of dynamic behavior of partially composite beams. In the available information about dynamic behavior of partially composite beams, the longitudinal inertia of motion has been neglected a priori. In the opinion of the authors, this is inherited from dynamic analysis of uniform beams to acquire mathematical convenience. Otherwise, analytical solutions of dynamic equations of partially composite beams would be rather difficult to develop due to the coupling of flexural and axial motions.

In this paper, the weak-form quadrature element method is applied to static and free vibrational analyses of partially composite beams. The weak-form quadrature element method (QEM) [11-14] has been shown to be rather successful in improving computational efficiency. So far, the QEM has been applied to various structural problems [11-14]. Representative cases with various levels of shear connection stiffness are studied in this paper and the results are compared with those of finite element formulations [10]. The rapid convergence, high accuracy, and slip-locking-free characteristics of the weak-form quadrature element method are highlighted. In addition, free vibration analysis of partially composite beams is conducted with the longitudinal inertia of motion included. It is shown that the longitudinal inertia of motion cannot be simply neglected since low-order frequencies may be significantly affected.

\section{Kinematic Model and Quadrature Element Formulation}

In this section, quadrature element formulation will be derived based on Newmark kinematical model.

\subsection{Newmark Kinematical Model}

For simplicity, partially composite beams are modeled as two sub-Euler-Bernoulli beams linked by a deformable connection which allows for relative axial displacement $\Delta u$ between 


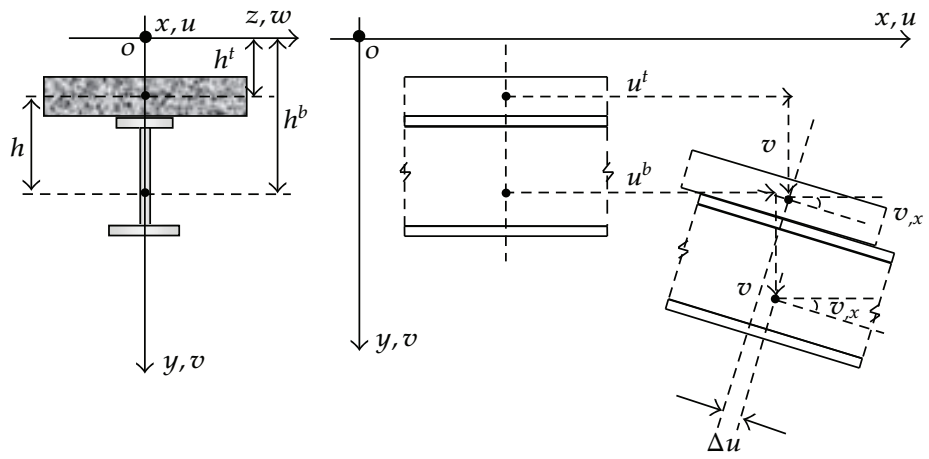

Figure 1: Newmark kinematical model and displacement field for a partially composite beam.

the two layers without transverse separation [1]. The assumption of small displacements, rotations, and strains is made for the two layers. In this paper, planar flexural and free vibrational analyses of partially composite beams are considered.

A typical composite beam with a symmetric cross-section is shown in Figure 1, where the top subcomponent is formed by a concrete slab while the bottom subcomponent is formed by a steel joist. Without losing generality, the formulation is derived for a beam segment of length $L$ and about an arbitrary reference axis $x$ located at distances $h^{t}$ and $h^{b}$ from the centroids of two subcomponents, respectively.

The displacement field of the partially composite beam consists of transverse displacement $v(x, t)$, axial displacements $u^{t}(x, t)$ and $u^{b}(x, t)$ at the centroids of the top and bottom sub-components. For simplicity, these displacements will be abbreviated as $v, u^{t}$, and $u^{b}$. The expressions for the rotation and the curvature at a cross-section of the beam, denoted as $v_{, x}, v_{, x x}$, respectively, can be obtained by differentiating the deflection with respect to coordinate $x$.

Under the assumptions of Bernoulli-Euler beams, only axial strains exist, that is,

$$
\varepsilon_{x}^{t}=u_{, x}^{t}-\left(y-h^{t}\right) v_{, x x} \quad \varepsilon_{x}^{b}=u_{, x}^{b}-\left(y-h^{b}\right) v_{, x x},
$$

while the slip at the interface is expressed by the following relation:

$$
\Delta u=u^{b}-u^{t}+h v_{, x}
$$

where $h=h^{b}-h^{t}$ is the distance between the two subcomponent centroids (Figure 1). The parameters describing the strains can be collected in a vector:

$$
\varepsilon=\left[\begin{array}{lll}
u_{, x}^{t} & u_{, x}^{b}-v_{, x x}
\end{array}\right]^{T},
$$

whose corresponding internal forces can be expressed in a vector as

$$
\boldsymbol{\sigma}=\left[\begin{array}{lll}
N^{t} & N^{b} & M
\end{array}\right]^{T}
$$


with the axial forces in two subcomponents $N^{t}, N^{b}$ and the summation of the bending moments of two subcomponents $M$. Then, the linear generalized constitutive relation between strains and internal forces can be written in the form:

$$
\sigma=\mathrm{E} \varepsilon
$$

The elasticity matrix is

$$
\mathbf{E}=\left[\begin{array}{ccc}
E^{t} A^{t} & 0 & 0 \\
0 & E^{b} A^{b} & 0 \\
0 & 0 & E^{t} I^{t}+E^{b} I^{b}
\end{array}\right]
$$

where $A^{t}, I^{t}$, and $E^{t}$ are the cross-section area, the second moment of area about the neutral axis and the elastic modulus of the material of the top subcomponent; symbols with superscript $b$ designate relevant properties of the bottom subcomponent. The interface shear force can be written as

$$
f_{s}=K \Delta u,
$$

where $K$ is the shear connection stiffness.

\subsection{Quadrature Element Formulation}

In quadrature element analysis, the following normalized dimensionless coordinate is introduced in a quadrature-beam element:

$$
\xi=\frac{2}{L} x-1, \quad \xi \in[-1,1]
$$

The strain energy of the element is then written as

$$
\begin{aligned}
U^{(e)} & =\int_{0}^{L} \frac{1}{2}\left(\boldsymbol{\varepsilon}^{T} \mathbf{E} \boldsymbol{\varepsilon}+\Delta u K \Delta u\right) d x=\int_{0}^{L} \frac{1}{2}\left(\boldsymbol{\varepsilon}^{T} \mathbf{E} \boldsymbol{\varepsilon}+\mathbf{u}^{T} \mathbf{E}_{s} \mathbf{u}\right) d x \\
& =\int_{-1}^{1} \frac{1}{2}\left(\overline{\boldsymbol{\varepsilon}}^{T} \mathbf{D}^{T} \mathbf{E} \mathbf{D} \overline{\boldsymbol{\varepsilon}}+\overline{\mathbf{u}}^{T} \mathbf{D}_{s}^{T} \mathbf{E}_{s} \mathbf{D}_{s} \overline{\mathbf{u}}\right) \frac{L}{2} d \xi
\end{aligned}
$$

where

$$
\boldsymbol{\varepsilon}=\left[\begin{array}{lll}
u^{t}, x & u^{b}, x & -v_{, x x}
\end{array}\right]^{T}=\mathbf{D} \bar{\varepsilon},
$$

with

$$
\mathbf{D}=\left[\begin{array}{ccc}
2 / L & 0 & 0 \\
0 & 2 / L & 0 \\
0 & 0 & -4 / L^{2}
\end{array}\right], \quad \bar{\varepsilon}=\left[\begin{array}{lll}
u_{, \xi}^{t} & u_{, \xi}^{b} & v_{, \xi \xi}
\end{array}\right]^{T}
$$


The displacement vector for the interlayer slip is defined as

$$
\mathbf{u}=\left[\begin{array}{lll}
u^{t} & u^{b} & v_{, x}
\end{array}\right]^{T}=\mathbf{D}_{s} \overline{\mathbf{u}}
$$

with

$$
\mathbf{D}_{s}=\left[\begin{array}{ccc}
1 & 0 & 0 \\
0 & 1 & 0 \\
0 & 0 & 2 / L
\end{array}\right], \quad \overline{\mathbf{u}}=\left\{\begin{array}{lll}
u^{t} & u^{b} & v, \xi
\end{array}\right\}^{T}
$$

The interlayer elasticity matrix is given by

$$
\mathbf{E}_{s}=K\left[\begin{array}{ccc}
1 & -1 & -h \\
-1 & 1 & h \\
-h & h & h^{2}
\end{array}\right]
$$

It is noted that quantities with an overbar are designated as variables in the normalized coordinate system.

An efficient numerical scheme is chosen first to evaluate the strain energy. Thus, (2.9) can be rewritten as

$$
U^{(e)}=\frac{1}{2} \sum_{i=1}^{N} w_{i}\left(\overline{\boldsymbol{\varepsilon}}_{i}^{T} \mathbf{D}^{T} \mathbf{E}_{i} \mathbf{D} \overline{\boldsymbol{\varepsilon}}_{i}+\overline{\mathbf{u}}_{i}^{T} \mathbf{D}_{s}^{T} \mathbf{E}_{s i} \mathbf{D}_{s} \overline{\mathbf{u}}_{i}\right) \frac{L}{2},
$$

where $w_{i}$ denotes the weighting coefficients in the numerical integration scheme. Usually, Lobatto rule [19] is employed in a quadrature element whose sampling point distribution in the normalized domain is

$$
\xi_{1}=-1, \ldots, \xi_{i}, \ldots, \xi_{N}=-1 \quad i=2 \cdots N-1,
$$

where $\xi_{i}$ is the $(i-1)$ th zero of the first-order derivative of the $(N-1)$ th-order Legendre polynomial $P_{N-1}(\xi)$.

Denote the slopes at the two ends of the beam as $\theta_{1}$ and $\theta_{N}$ and note that

$$
\theta_{1}=\left(v_{, x}\right)_{1}, \quad \theta_{N}=\left(v_{, x}\right)_{N}
$$

The nodal displacement vector of the quadrature element in the physical domain is written as (see Figure 2)

$$
\begin{aligned}
\mathbf{d}^{(e)}=\left\{\begin{array}{lllllllll}
\theta_{1} & u_{1}^{t} & u_{1}^{b} & v_{1} & u_{2}^{t} & u_{2}^{b} & v_{2} & \cdots & \\
u_{N-1}^{t} & u_{N-1}^{b} & v_{N-1} & u_{N}^{t} & u_{N}^{b} & v_{N} & \theta_{N}
\end{array}\right\}^{T},
\end{aligned}
$$




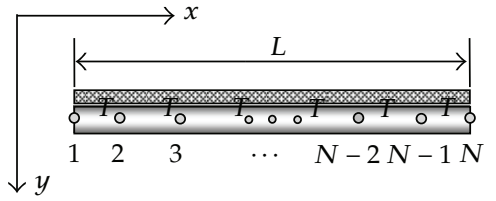

(a) Physical coordinate system

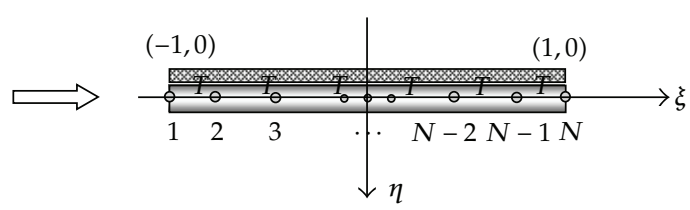

(b) Normalized coordinate system

Figure 2: Discretization and coordinate systems of a quadrature element.

which is related to its counterpart in the normalized dimensionless coordinate system through

$$
\overline{\mathbf{d}}^{(e)}=\mathbf{T d}^{(e)},
$$

where

$$
\begin{aligned}
& \overline{\mathbf{d}}^{(e)}=\left\{\begin{array}{llllllll}
\left(v_{, \xi}\right)_{1} & u_{1}^{t} & u_{1}^{b} & v_{1} & u_{2}^{t} & v_{2}^{b} & v_{2} & \cdots
\end{array}\right. \\
& \left.u_{N-1}^{t} \quad u_{N-1}^{b} \quad v_{N-1} \quad u_{N}^{t} \quad u_{N}^{b} \quad v_{N}\left(v_{, \xi}\right)_{N}\right\}^{T}, \\
& \mathbf{T}=\operatorname{diag}\left(\frac{L}{2}, 1, \ldots, 1, \frac{L}{2}\right) \text {. }
\end{aligned}
$$

With differential quadrature analogs [20], the normalized strain at a node (integration point) in (2.10) can be related to the normalized nodal displacement vector as

$$
\bar{\varepsilon}_{i}=\mathbf{B}_{i} \overline{\mathbf{d}}^{(e)}
$$

Similarly, the normalized displacement vector for the interlayer slip can also be expressed in terms of the normalized nodal displacement vector as

$$
\overline{\mathbf{u}}_{i}=\mathbf{B}_{s i} \overline{\mathbf{d}}^{(e)}
$$

The essence of the conventional differential quadrature analog is that the derivative of a function at a node is expressed as weighted linear summation of function values at all nodes in the domain of interest [20].

Symbolically, the $m$ th-order derivative of a function $f$ with respect to a variable $\xi$ at a node $\xi=\xi_{i}$ is approximated by

$$
\left.\frac{d^{m} f}{d \xi^{m}}\right|_{\xi=\xi_{i}}=\sum_{j=1}^{N} C_{i j}^{(m)} f\left(\xi_{j}\right)
$$


where $C_{i j}^{(m)}$ are the weighting coefficients for $m$ th-order derivatives determined by using Lagrange interpolation functions as basis functions [21] and $f\left(\xi_{j}\right)$ is function value at node $\xi=\xi_{j}$. Details of differential quadrature analogs can be referred to $[22,23]$.

For Bernoulli-Euler beams, an element end condition necessitates the consideration of the slope as well as the deflection of the beam. Thus, a generalized differential quadrature analog is introduced as

$$
\left.\frac{d^{m} f}{d \xi^{m}}\right|_{\xi=\xi_{i}}=\left.G_{i 1}^{(m)} \frac{d f}{d \xi}\right|_{\xi=\xi_{1}}+\sum_{j=1}^{N} G_{i(j+1)}^{(m)} f\left(\xi_{j}\right)+\left.G_{i(N+2)}^{(m)} \frac{d f}{d \xi}\right|_{\xi=\xi_{N}},
$$

where $G_{i j}^{(m)}$ are weighting coefficients for $m$ th order derivatives in the generalized differential quadrature analog and determined by using Hermitian interpolation functions as basis functions [13]. All derivatives involved in (2.11) and (2.13) are approximated using the differential quadrature analogs given in (2.23) and (2.24), that is,

$$
\begin{gathered}
\left(u_{, \xi}^{t}\right)_{i}=\sum_{j=1}^{N} C_{i j}^{(1)} u_{j}^{t}, \quad\left(u^{b}, \xi\right)_{i}=\sum_{j=1}^{N} C_{i j}^{(1)} u_{j}^{b}, \\
\left(v_{, \xi}\right)_{i}=G_{i 1}^{(1)} v_{1, \xi}+\sum_{j=1}^{N} G_{i(j+1)}^{(1)} v_{j}+G_{i(N+2)}^{(1)} v_{N, \xi}, \\
\left(v_{, \xi \xi}\right)_{i}=G_{i 1}^{(2)} v_{1, \xi}+\sum_{j=1}^{N} G_{i(j+1)}^{(2)} v_{j}+G_{i(N+2)}^{(2)} v_{N, \xi}
\end{gathered}
$$

Substitution of (2.25) into (2.21) and (2.22) yields the expressions of $\mathbf{B}_{i}$ and $\mathbf{B}_{s i}$, that is,

$$
\begin{aligned}
\mathbf{B}_{i} & =\left[\begin{array}{c|c|ccc|c|c}
0 & \cdots & C_{i j}^{(1)} & 0 & 0 & \cdots & 0 \\
0 & \cdots & 0 & C_{i j}^{(1)} & 0 & \cdots & 0 \\
G_{i 1}^{(2)} & \cdots & 0 & 0 & G_{i(j+1)}^{(2)} & \cdots & G_{i(N+2)}^{(2)}
\end{array}\right] \quad j=1, \ldots, N, \\
\mathbf{B}_{s i} & =\left[\begin{array}{c|c|ccc|c|c}
0 & \cdots & \delta_{i j} & 0 & 0 & \cdots & 0 \\
0 & \cdots & 0 & \delta_{i j} & 0 & \cdots & 0 \\
G_{i 1}^{(1)} & \cdots & 0 & 0 & G_{i(j+1)}^{(1)} & \cdots & G_{i(N+2)}^{(1)}
\end{array}\right] \quad j=1, \ldots, N,
\end{aligned}
$$

where $\delta_{i j}$ is the Kronecker delta, implying

$$
\delta_{i j}= \begin{cases}1, & i=j \\ 0, & \text { otherwise }\end{cases}
$$

Then, (2.15) is rewritten as

$$
U^{(e)}=\frac{1}{2} \overline{\mathbf{d}}^{(e) T} \overline{\mathbf{K}}^{(e)}
$$


where

$$
\overline{\mathbf{K}}=\sum_{i=1}^{N} w_{i}\left(\mathbf{B}_{i}^{T} \mathbf{D}^{T} \mathbf{E}_{i} \mathbf{D} \mathbf{B}_{i}+\mathbf{B}_{s i}^{T} \mathbf{D}_{s}^{T} \mathbf{E}_{s i} \mathbf{D}_{s} \mathbf{B}_{s i}\right) \frac{L}{2} .
$$

The kinetic energy of the partially composite beam consists of three portions: longitudinal kinetic energy, flexural energy, and rotational energy which are given, respectively, by

$$
\begin{gathered}
T_{u}^{(e)}=\frac{1}{2} \int_{-1}^{1}\left[\rho^{t} A^{t}\left(\dot{u}^{t}\right)^{2}+\rho^{b} A^{b}\left(\dot{u}^{b}\right)^{2}\right] \frac{L}{2} d \xi, \\
T_{v}^{(e)}=\frac{1}{2} \int_{-1}^{1}\left[\left(\rho^{t} A^{t}+\rho^{b} A^{b}\right) \dot{v}^{2}\right] \frac{L}{2} d \xi \\
T_{r}^{(e)}=\frac{1}{2} \int_{-1}^{1}\left[\frac{4}{L^{2}}\left(\rho^{t} I^{t}+\rho^{b} I^{b}\right) \dot{v}_{, \xi}^{2}\right] \frac{L}{2} d \xi
\end{gathered}
$$

where the single dot stands for the first-order partial derivative with respect to time variable. Introduction of the same numerical integration and the normalized nodal displacement vector turns the kinetic energy of the beam into

$$
\begin{aligned}
T^{(e)} & =T_{u}^{(e)}+T_{v}^{(e)}+T_{r}^{(e)} \\
& =\frac{1}{2} \dot{\overline{\mathbf{d}}}^{(e) T}\left(\sum_{i=1}^{N} w_{i} \mathbf{B}_{d i}^{T} \boldsymbol{\Lambda}_{i} \mathbf{B}_{d i} \frac{L}{2}\right) \dot{\overline{\mathbf{d}}}^{(e)} \\
& =\frac{1}{2} \dot{\overline{\mathbf{d}}}^{(e) T} \overline{\mathbf{M}}^{(e)} \dot{\overline{\mathbf{d}}}^{(e)},
\end{aligned}
$$

where

$$
\overline{\mathbf{M}}^{(e)}=\sum_{i=1}^{N} w_{i} \mathbf{B}_{d i}^{T} \mathbf{\Lambda}_{i} \mathbf{B}_{d i} \frac{L}{2}
$$

with

$$
\begin{gathered}
\mathbf{B}_{d i}=\left[\begin{array}{c|c|ccc|c|c}
0 & \cdots & \delta_{i j} & 0 & 0 & \cdots & 0 \\
0 & \cdots & 0 & \delta_{i j} & 0 & \cdots & 0 \\
0 & \cdots & 0 & 0 & \delta_{i j} & \cdots & 0 \\
G_{i 1}^{(1)} & \cdots & 0 & 0 & G_{i(j+1)}^{(1)} & \cdots & G_{i(N+2)}^{(1)}
\end{array}\right], \quad j=1, \ldots, N \\
\Lambda=\left[\begin{array}{cccc}
\rho^{t} A^{t} & 0 & 0 & 0 \\
0 & \rho^{b} A^{b} & 0 & 0 \\
0 & 0 & \rho^{t} A^{t}+\rho^{b} A^{b} & 0 \\
0 & 0 & 0 & \frac{4}{L^{2}}\left(\rho^{t} I^{t}+\rho^{b} I^{b}\right)
\end{array}\right] .
\end{gathered}
$$




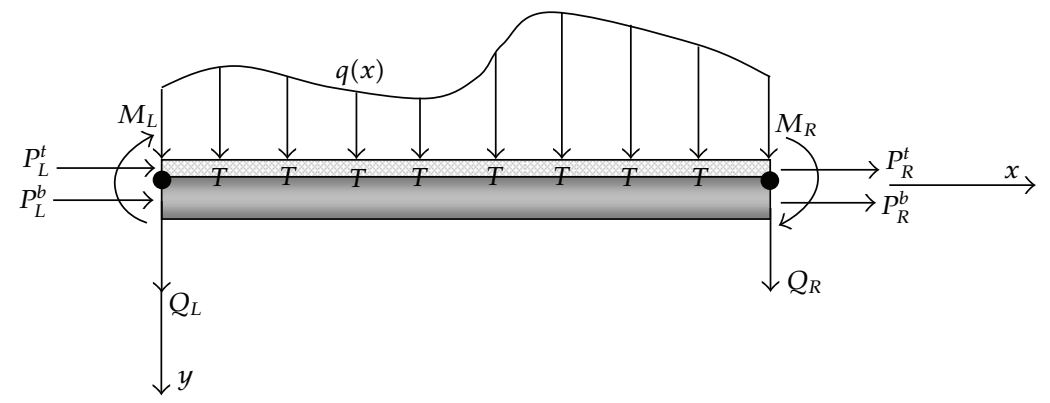

Figure 3: Forces applied on an element in physical coordinate system.

The energy expression of external forces (see Figure 3) applied on the beam element is

$$
\begin{aligned}
V^{(e)}= & -\int_{0}^{L} q(x) v(x) d x-P_{L}^{t} u_{L}^{t}-P_{L}^{b} u_{L}^{b}-P_{R}^{t} u_{R}^{t}-P_{R}^{b} u_{R}^{b} \\
& -Q_{L} v_{L}-Q_{R} v_{R}-M_{L} \theta_{L}-M_{R} \theta_{R} \\
= & -\int_{-1}^{+1} q(\xi) v(\xi) \frac{L}{2} d \xi-P_{L}^{t} u_{L}^{t}-P_{L}^{b} u_{L}^{b}-P_{R}^{t} u_{R}^{t}-P_{R}^{b} u_{R}^{b} \\
& -Q_{L} v_{L}-Q_{R} v_{R}-\frac{2}{L} M_{L} v_{L, \xi}-\frac{2}{L} M_{R} v_{R, \xi} \\
= & -\overline{\mathbf{d}}^{(e) T} \overline{\mathbf{F}}^{(e)} .
\end{aligned}
$$

The same numerical integration scheme was introduced into (2.34), where $\overline{\mathbf{F}}^{(e)}$ is the element force vector given by

$$
\begin{aligned}
\overline{\mathbf{F}}^{(e)}=\left\{\begin{array}{lllllllll}
f_{v 1}^{\prime} & f_{u 1}^{t} & f_{u 1}^{b} & f_{v 1} & f_{u 2}^{t} & f_{u 2}^{b} & f_{v 2} & \cdots & \\
& f_{u N-1}^{\mathrm{t}} & f_{u N-1}^{\mathrm{b}} & f_{v N-1} & f_{u N}^{\mathrm{t}} & f_{u N}^{\mathrm{b}} & f_{v N} & f_{v N}^{\prime}
\end{array}\right\}
\end{aligned}
$$

with

$$
\begin{gathered}
f_{v 1}^{\prime}=\frac{2}{L} M_{L}, \quad f_{u 1}^{t}=P_{L^{\prime}}^{t} \quad f_{u 1}^{b}=P_{L^{\prime}}^{b} \quad f_{v 1}=\frac{L}{2} w_{1} q\left(\xi_{1}\right)+Q_{L} ; \\
f_{u 2}^{t}=0, \quad f_{u 2}^{b}=0, \quad f_{v 2}^{b}=\frac{L}{2} w_{2} q\left(\xi_{2}\right) ; \\
\vdots \quad \\
f_{u N-1}^{t}=0, \quad f_{u N-1}^{b}=0, \quad f_{v N-1}=\frac{L}{2} w_{N-1} q\left(\xi_{N-1}\right) ; \\
f_{u N}^{t}=P_{R^{\prime}}^{t} \quad f_{u N}^{b}=P_{R^{\prime}}^{b} \quad f_{v N}=\frac{L}{2} w_{N} q\left(\xi_{N}\right)+Q_{R}, \quad f_{v N}^{\prime}=\frac{2}{L} M_{R} .
\end{gathered}
$$


Hamilton principle dictates the following condition of the element:

$$
\delta \int_{t_{1}}^{t_{2}}\left(T^{(e)}-U^{(e)}-V^{(e)}\right) d t=0,
$$

where $t_{1}$ and $t_{2}$ are two instants of time. Substitution of (2.28), (2.31), and (2.34) into (2.37) yields

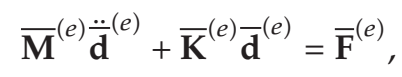

where the double dot represents the second-order differentiation with respect to time variable. Introducing the coordinate transformation (see (2.19)), the element equilibrium equations in physical domain become

$$
\mathbf{M}^{(e)} \ddot{\mathbf{d}}(e)+\mathbf{K}^{(e)} \mathbf{d}^{(e)}=\mathbf{F}^{(e)},
$$

where

$$
\mathbf{K}^{(e)}=\mathbf{T} \overline{\mathbf{K}}^{(e)} \mathbf{T}, \quad \mathbf{M}^{(e)}=\mathbf{T} \overline{\mathbf{M}}^{(e)} \mathbf{T}, \quad \mathbf{F}^{(e)}=\mathbf{T} \overline{\mathbf{F}}^{(e)}, \quad \mathbf{d}^{(e)}=\mathbf{T}^{-1} \overline{\mathbf{d}}^{(e)} .
$$

Applying Hamilton principle to the entire beam yields the global dynamic equilibrium equations of the system

$$
\mathbf{M} \ddot{\mathbf{d}}+\mathbf{K d}=\mathbf{F},
$$

where $\mathbf{d}$ is the global nodal displacement vector; $\mathbf{K}, \mathbf{M}$, and $\mathbf{F}$ are the global stiffness matrix, the global mass matrix, and the global force vector, respectively.

There are three typical boundary conditions that are usually considered in analysis of composite beams: clamped end (C), simply supported end (S), and free end (F). For a clamped end, the following conditions are enforced:

$$
u^{t}=u^{b}=v=v_{, x}=0 .
$$

For a simply supported end, it is required that

$$
u^{b}=v=0
$$

The displacement variables or internal forces at an arbitrary position can be obtained using Langrange interpolation and differential quadrature analogs after solving for the nodal displacements in (2.41). 


\section{Numerical Results}

To illustrate the effectiveness of the QEM in analysis of partially composite beams, examples are studied in this section.

\subsection{Flexural Analysis}

In static analysis, neglecting the inertial terms, (2.41) is reduced to

$$
\mathbf{K d}=\mathbf{F} \text {. }
$$

For comparison, a typical partially composite beam reported in [9] is re-examined firstly. The partially composite beam of 20 meters in length $(L)$ is subjected to a uniformly distributed load of $35 \mathrm{kN} / \mathrm{m}$. The two subcomponents of the beam are a rectangular reinforced concrete slab $(2300 \mathrm{~mm} \times 230 \mathrm{~mm})$ with $1 \%$ reinforcement and steel joist with an I-shaped crosssection. The steel joist has both flanges of $500 \mathrm{~mm} \times 40 \mathrm{~mm}$ and a web of $1120 \mathrm{~mm} \times 16 \mathrm{~mm}$. The elastic modulus for the steel reinforcement and joist is $210,000 \mathrm{MPa}$ while the elastic modulus for the concrete is $34,200 \mathrm{MPa}$. The densities of the two materials are $2400 \mathrm{Kg} / \mathrm{m}^{3}$

and $7850 \mathrm{Kg} / \mathrm{m}^{3}$, respectively. The beam with various levels of shear connection is considered to evaluate the proposed quadrature element formulation for $\alpha L$ varying from 1 to 40 where $\alpha L$ is the dimensionless shear stiffness coefficient first identified by Girhammar and Pan [2] and defined as

$$
\alpha L=\sqrt{K\left(\frac{1}{E^{t} A^{t}}+\frac{1}{E^{b} A^{b}}+\frac{h^{2}}{E^{t} I^{t}+E^{b} I^{b}}\right)} L .
$$

The chosen values of $\alpha L$ in the paper are as typical as those used in many other references [8-10].

For brevity, two capital letters which stand for the boundary conditions at the two ends of the beam are used to designate the beam. Thus, four typical boundary conditions of the beam: SS, FC, SC, and CC are considered in the present investigation.

The relative errors of the maximum deflection and the maximum slip in the beam are defined as

$$
\begin{gathered}
v_{r e}=\left|\frac{v_{\max }-\left(v_{\max }\right)_{\text {exact }}}{\left(v_{\max }\right)_{\text {exact }}}\right|, \\
\Delta u_{r e}=\left|\frac{\Delta u_{\max }-\left(\Delta u_{\max }\right)_{\text {exact }}}{\left(\Delta u_{\max }\right)_{\text {exact }}}\right|,
\end{gathered}
$$

respectively. The exact values of the two displacements in (3.3) under four boundary conditions are computed using the exact solution given in [2].

The computed results of the maximum deflection for various shear connection stiffnesses are listed and compared with finite element solutions in Tables 1, 2, 3, and 4. Acronyms are used to refer to the results for various formulations and discretizations. For instance, 1Q8.26DOF is used to designate one quadrature element with 8 nodes and 26 total degrees of freedom; 4F8.20DOF stands for four finite elements, each with eight degrees of 
Table 1: Relative percentage error of maximum deflection of simply supported beam.

\begin{tabular}{cccccccc}
\hline & & \multicolumn{7}{c}{$\alpha L$} \\
& & 1 & 5 & 10 & 20 & 30 & 40 \\
\hline \multirow{3}{*}{ QEM } & 1Q5.17DOF & 0.0115 & 0.0828 & 0.0526 & 0.0109 & 0.0029 & 0.0011 \\
& 1Q8.26DOF & 0.0000 & 0.0009 & 0.0026 & 0.0020 & 0.0010 & 0.0005 \\
& 1Q12.38DOF & 0.0000 & 0.0000 & 0.0000 & 0.0000 & 0.0001 & 0.0001 \\
\hline \multirow{4}{*}{ FEM } & 4F8.20DOF & 0.0088 & 1.0530 & 2.1147 & 2.9013 & 3.3907 & 3.7683 \\
& 8F8.36DOF & 0.0027 & 0.2639 & 0.5094 & 0.6417 & 0.7004 & 0.7518 \\
& 4F10.28DOF & 0.0024 & 0.0076 & 0.0020 & 0.0002 & 0.0001 & 0.0001 \\
& 8F10.52DOF & 0.0001 & 0.0004 & 0.0001 & 0.0000 & 0.0000 & 0.0000 \\
& 2F16.28DOF & 0.0000 & 0.0001 & 0.0008 & 0.0009 & 0.0005 & 0.0003 \\
& 4F16.52DOF & 0.0000 & 0.0000 & 0.0000 & 0.0000 & 0.0000 & 0.0000 \\
\hline
\end{tabular}

Table 2: Relative percentage error of maximum deflection of cantilever beam.

\begin{tabular}{cccccccc}
\hline & & \multicolumn{7}{c}{$\alpha L$} \\
& & 1 & 5 & 10 & 20 & 30 & 40 \\
\hline \multirow{3}{*}{ QEM } & 1Q5.14DOF & 0.0039 & 0.1770 & 0.1861 & 0.0953 & 0.0532 & 0.0358 \\
& 1Q8.26DOF & 0.0000 & 0.0000 & 0.0001 & 0.0010 & 0.0017 & 0.0042 \\
& 1Q12.38DOF & 0.0000 & 0.0000 & 0.0000 & 0.0000 & 0.0000 & 0.0024 \\
\hline \multirow{4}{*}{ FEM } & 4F8.20DOF & 0.0310 & 0.5560 & 0.8422 & 1.1587 & 1.3873 & 1.5596 \\
& 8F8.36DOF & 0.0076 & 0.1361 & 0.1981 & 0.2465 & 0.2768 & 0.3060 \\
& 4F10.28DOF & 0.0011 & 0.0093 & 0.0140 & 0.0127 & 0.0094 & 0.0093 \\
& 8F10.52DOF & 0.0001 & 0.0006 & 0.0012 & 0.0018 & 0.0018 & 0.0040 \\
& 2F16.28DOF & 0.0000 & 0.0001 & 0.0009 & 0.0028 & 0.0032 & 0.0053 \\
& 4F16.52DOF & 0.0000 & 0.0000 & 0.0000 & 0.0001 & 0.0003 & 0.0028 \\
\hline
\end{tabular}

freedom, and 20 degrees of freedom in total. The nodes in an element are equally spaced for all the finite elements as proposed by Dall'Asta and Zona [10] and the beam is divided into elements of the same size.

From Tables 1-4, it is seen that both the quadrature element and finite element formulations are able to provide a good estimate of the maximum deflection under various levels of connection stiffness. It is found that quadrature element formulation offers high computational efficiency than finite element formulation does, especially for large shear connection stiffness, say, $\alpha L>20$. For instance, the error of one four-node quadrature element with $14 \mathrm{DOF}$ in total is less than that of eight 8DOF finite elements with 36DOF in total. It is observed that given the same number of degrees of freedom, the relative error of the maximum deflection is in the following ascending sequence for the four boundary conditions: SS, FC, SC and CC in example one. This is believed to be ascribed to the high-order feature due to the enhancement of constraints at the two ends of the composite beam.

The computed results of the maximum slip for various shear connection stiffnesses of the two examples are listed and compared with the finite element solutions in Tables $5,6,7$, and 8 . For computation of the slip, low-order finite element solutions have been 
Table 3: Relative percentage error of maximum deflection of propped cantilever beam.

\begin{tabular}{cccccccc}
\hline & & \multicolumn{7}{c}{$\alpha L$} \\
& & 1 & 5 & 10 & 20 & 30 & 40 \\
\hline \multirow{3}{*}{ QEM } & 1Q5.17DOF & 0.0412 & 0.4503 & 0.3315 & 0.0171 & 0.0397 & 0.0426 \\
& 1Q8.26DOF & 0.0000 & 0.0012 & 0.0035 & 0.0068 & 0.0111 & 0.0125 \\
& 1Q12.38DOF & 0.0000 & 0.0000 & 0.0000 & 0.0004 & 0.0010 & 0.0011 \\
\hline \multirow{4}{*}{ FEM } & 4F8.20DOF & 0.1351 & 1.2135 & 3.8055 & 7.0272 & 8.9315 & 10.1849 \\
& 8F8.36DOF & 0.0120 & 0.2815 & 0.8672 & 1.4473 & 1.7387 & 1.9599 \\
& 4F10.28DOF & 0.1297 & 0.1400 & 0.2065 & 0.2284 & 0.2079 & 0.1895 \\
& 8F10.52DOF & 0.0106 & 0.0114 & 0.0178 & 0.0249 & 0.0257 & 0.0243 \\
& 2F16.28DOF & 0.0003 & 0.0040 & 0.0091 & 0.0230 & 0.0279 & 0.0262 \\
& 4F16.52DOF & 0.0000 & 0.0000 & 0.0001 & 0.0010 & 0.0022 & 0.0030 \\
\hline
\end{tabular}

Table 4: Relative percentage error of maximum deflection of clamped-clamped beam.

\begin{tabular}{cccccccc}
\hline & & \multicolumn{6}{c}{$\alpha L$} \\
& & 1 & 5 & 10 & 20 & 30 & 40 \\
\hline \multirow{3}{*}{ QEM } & 1Q5.17DOF & 0.0205 & 0.3513 & 0.4922 & 0.1474 & 0.0024 & 0.0404 \\
& 1Q8.26DOF & 0.0000 & 0.0003 & 0.0063 & 0.0230 & 0.0180 & 0.0080 \\
& 1Q12.38DOF & 0.0000 & 0.0000 & 0.0001 & 0.0022 & 0.0052 & 0.0070 \\
\hline \multirow{4}{*}{ FEM } & 4F8.20DOF & 0.0031 & 1.0009 & 4.8362 & 11.4867 & 15.7132 & 18.4493 \\
& 8F8.36DOF & 0.0008 & 0.2397 & 1.0726 & 2.2468 & 2.9022 & 3.3890 \\
& 4F10.28DOF & 0.0002 & 0.0601 & 0.2198 & 0.2666 & 0.2029 & 0.1503 \\
& 8F10.52DOF & 0.0000 & 0.0049 & 0.0211 & 0.0394 & 0.0413 & 0.0375 \\
& 2F16.28DOF & 0.0000 & 0.0000 & 0.0018 & 0.0180 & 0.0318 & 0.0357 \\
& 4F16.52DOF & 0.0000 & 0.0000 & 0.0002 & 0.0026 & 0.0060 & 0.0083 \\
\hline
\end{tabular}

afflicted with large error for partially composite beams with large shear connection stiffness regardless of the end conditions. This phenomenon has been known as "slip locking." In order to overcome the problem, finite elements with more degrees of freedom were proposed [10] by introducing additional internal nodes into low-order finite elements. From Tables 5-8, it is observed that the accuracy of slip for beams with high shear connection stiffness using high-order finite elements indeed improves. In contrast, quadrature element solutions of the slip are less affected by the sliplocking especially for elements with large $N$.

It is seen that the quadrature element solution of the relative error of the maximum slip fluctuates slightly with the increase of shear connection stiffness. Given the same number of DOF, large shear connection stiffness in general results in large maximum slip error and the largest relative error of the maximum slip occurs in the SC beam. For partially composite beams with large shear connection stiffness, say, $\alpha L \geq 40$, the number of integration points in a quadrature element has to exceed twelve to keep the relative error of the maximum slip below $1.0 \%$.

It is noted that the change of shear connection stiffness has significant influence on the variation of slip. The variation of slip along the beam length for a partially composite SC beam is given in Figure 4 for $\alpha L=10$ and $\alpha L=40$. In general, quadrature element 


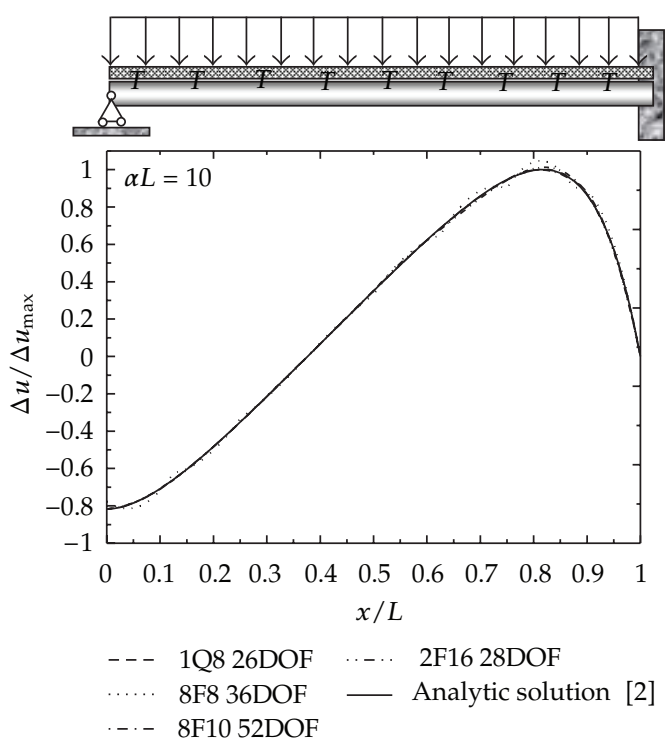

(a) $\alpha L=10$

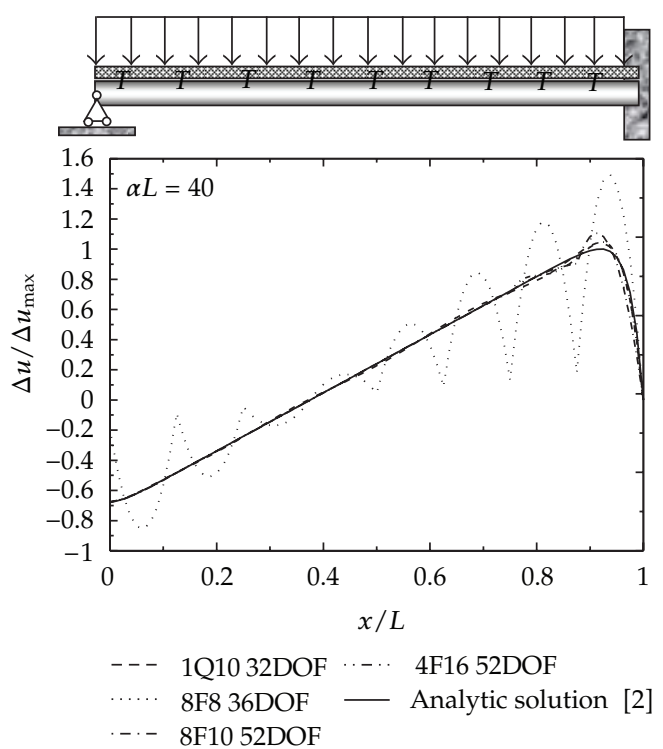

(b) $\alpha L=40$

Figure 4: Slip in propped cantilever beam.

Table 5: Relative percentage error of maximum slip of simply supported beam.

\begin{tabular}{cccccccc}
\hline & & \multicolumn{7}{c}{$\alpha L$} & & \\
& & 1 & 5 & 10 & 20 & 30 & 40 \\
\hline \multirow{3}{*}{ QEM } & 1Q5.17DOF & 0.0000 & 0.1351 & 1.0759 & 2.7046 & 2.9966 & 2.8370 \\
& 1Q8.26DOF & 0.0000 & 0.0000 & 0.0027 & 0.1039 & 0.3600 & 0.6232 \\
& 1Q12.38DOF & 0.0000 & 0.0000 & 0.0000 & 0.0001 & 0.0031 & 0.0170 \\
\hline \multirow{4}{*}{ FEM } & 4F8.20DOF & 0.0155 & 4.0115 & 19.0258 & 66.2752 & 116.7706 & 158.9260 \\
& 8F8.36DOF & 0.0052 & 1.0611 & 5.2659 & 21.5323 & 45.6728 & 74.2945 \\
& 4F10.28DOF & 0.0061 & 0.1035 & 0.4081 & 1.1653 & 1.6049 & 1.7651 \\
& 8F10.52DOF & 0.0004 & 0.0071 & 0.0366 & 0.1810 & 0.3758 & 0.5519 \\
& 2F16.28DOF & 0.0000 & 0.0011 & 0.0277 & 0.2843 & 0.6536 & 0.9370 \\
& 4F16.52DOF & 0.0000 & 0.0000 & 0.0004 & 0.0122 & 0.0572 & 0.1338 \\
\hline
\end{tabular}

Table 6: Relative percentage error of maximum slip of cantilever beam.

\begin{tabular}{cccccccc}
\hline & & \multicolumn{7}{c}{$\alpha L$} & & \\
& & 1 & 5 & 10 & 20 & 30 & 40 \\
\hline \multirow{3}{*}{ QEM } & 1Q5.17DOF & 0.0117 & 1.9410 & 4.1008 & 1.6481 & 1.1657 & 3.7099 \\
& 1Q8.26DOF & 0.0000 & 0.0074 & 0.0553 & 2.3105 & 5.3586 & 6.3459 \\
& 1Q12.38DOF & 0.0000 & 0.0000 & 0.0005 & 0.0580 & 0.2907 & 0.2596 \\
\hline \multirow{4}{*}{ FEM } & 4F8.20DOF & 0.0200 & 4.0367 & 10.7055 & 50.6004 & 81.4855 & 101.8157 \\
& 8F8.36DOF & 0.0357 & 1.1951 & 3.3342 & 11.7254 & 24.8397 & 45.7010 \\
& 4F10.28DOF & 0.0174 & 0.4822 & 3.9348 & 10.4627 & 12.1420 & 11.5777 \\
& 8F10.52DOF & 0.0138 & 0.0445 & 0.2953 & 0.1168 & 6.3507 & 9.4574 \\
& 2F16.28DOF & 0.0003 & 0.1441 & 0.2973 & 4.9769 & 7.6101 & 8.1488 \\
& 4F16.52DOF & 0.0000 & 0.0025 & 0.0713 & 0.7681 & 1.1464 & 3.2393 \\
\hline
\end{tabular}


Table 7: Relative percentage error of maximum slip of propped cantilever beam.

\begin{tabular}{cccccccc}
\hline & & \multicolumn{7}{c}{$\alpha L$} \\
& & 1 & 5 & 10 & 20 & 30 & 40 \\
\hline \multirow{3}{*}{ QEM } & 1Q5.17DOF & 0.0001 & 1.6806 & 0.7554 & 5.6241 & 10.2234 & 13.1021 \\
& 1Q8.26DOF & 0.0000 & 0.0029 & 0.1323 & 2.7820 & 5.0270 & 5.7604 \\
& 1Q12.38DOF & 0.0000 & 0.0000 & 0.0000 & 0.0665 & 0.1166 & 1.0060 \\
\hline \multirow{4}{*}{ FEM } & 4F8.20DOF & 0.0047 & 3.4691 & 17.7471 & 53.4611 & 79.6958 & 96.9796 \\
& 8F8.36DOF & 0.0041 & 1.0268 & 4.7829 & 7.5776 & 28.6019 & 49.4699 \\
& 4F10.28DOF & 0.0147 & 1.8361 & 5.9860 & 9.2331 & 9.0984 & 8.3554 \\
& 8F10.52DOF & 0.0009 & 0.2300 & 0.0430 & 2.5204 & 7.3871 & 10.6871 \\
& 2F16.28DOF & 0.0000 & 0.0805 & 1.2552 & 5.1346 & 6.6773 & 6.8502 \\
& 4F16.52DOF & 0.0000 & 0.0083 & 0.0780 & 0.1981 & 2.1739 & 4.7962 \\
\hline
\end{tabular}

Table 8: Relative percentage error of maximum slip of clamped-clamped beam.

\begin{tabular}{cccccccc}
\hline & & \multicolumn{7}{c}{$\alpha L$} \\
& & 1 & 5 & 10 & 20 & 30 & 40 \\
\hline \multirow{3}{*}{ QEM } & 1Q5.17DOF & 0.0007 & 0.2305 & 0.6642 & 1.3308 & 4.6795 & 7.3614 \\
& 1Q8.26DOF & 0.0000 & 0.0028 & 0.1887 & 2.0347 & 3.4765 & 3.5457 \\
& 1Q12.38DOF & 0.0000 & 0.0000 & 0.0003 & 0.0337 & 0.1259 & 0.9194 \\
\hline \multirow{4}{*}{ FEM } & 4F8.20DOF & 1.6646 & 7.0916 & 21.0955 & 53.7003 & 76.5905 & 90.3888 \\
& 8F8.36DOF & 0.2509 & 1.8077 & 3.9901 & 10.1811 & 30.5908 & 50.6004 \\
& 4F10.28DOF & 1.5211 & 3.2731 & 6.1337 & 7.5444 & 6.5904 & 4.9291 \\
& 8F10.52DOF & 0.1760 & 0.1601 & 0.5474 & 3.9346 & 8.1057 & 10.4625 \\
& 2F16.28DOF & 0.0061 & 0.2749 & 1.6200 & 4.8982 & 5.6508 & 5.1854 \\
& 4F16.52DOF & 0.0001 & 0.0038 & 0.1436 & 0.2788 & 2.6331 & 4.9756 \\
\hline
\end{tabular}

Table 9: Relative percentage error of maximum deflection of two-span beam.

\begin{tabular}{cccccccc}
\hline & & \multicolumn{7}{c}{$\alpha L$} \\
& & 1 & 5 & 10 & 20 & 30 & 40 \\
\hline \multirow{3}{*}{ QEM } & 4Q3.32DOF & 2.2144 & 3.7193 & 3.7997 & 2.9423 & 2.5447 & 2.3587 \\
& 4Q5.56DOF & 0.0008 & 0.0120 & 0.0361 & 0.0405 & 0.0359 & 0.0282 \\
& 4Q8.92DOF & 0.0000 & 0.0000 & 0.0000 & 0.0009 & 0.0014 & 0.0013 \\
\hline \multirow{4}{*}{ FEM } & 8F8.36DOF & 0.0028 & 1.0639 & 3.7823 & 7.4336 & 9.5816 & 10.9917 \\
& 16F8.68DOF & 0.0010 & 0.2613 & 0.8665 & 1.5001 & 1.8529 & 2.0688 \\
& 8F10.52DOF & 0.0025 & 0.0045 & 0.0805 & 0.0712 & 0.0509 & 0.0358 \\
& 16F10.100DOF & 0.0004 & 0.0039 & 0.0000 & 0.0009 & 0.0006 & 0.0012 \\
& 4F16.52DOF & 0.0000 & 0.0028 & 0.0059 & 0.0084 & 0.0020 & 0.0012 \\
& 8F16.100DOF & 0.0000 & 0.0001 & 0.0007 & 0.0015 & 0.0014 & 0.0009 \\
\hline
\end{tabular}


Table 10: Relative percentage error of maximum slip of two-span beam.

\begin{tabular}{cccccccc}
\hline & & \multicolumn{7}{c}{$\alpha L$} & & & \\
& & 1 & 5 & 10 & 20 & 30 & 40 \\
\hline \multirow{3}{*}{ QEM } & 4Q3.32DOF & 0.0494 & 4.8837 & 7.2108 & 4.6493 & 2.2988 & 1.1436 \\
& 4Q5.56DOF & 0.0000 & 0.0152 & 0.1866 & 1.1148 & 3.6197 & 5.5888 \\
& 4Q8.92DOF & 0.0000 & 0.0001 & 0.0076 & 0.2123 & 0.8994 & 1.8320 \\
\hline \multirow{4}{*}{ FEM } & 8F8.36DOF & 0.0221 & 2.3294 & 8.7591 & 40.8025 & 77.7317 & 106.2524 \\
& 16F8.68DOF & 0.0056 & 1.0457 & 2.9914 & 12.0138 & 25.1913 & 40.8953 \\
& 8F10.52DOF & 0.0005 & 0.2944 & 0.8637 & 6.1695 & 11.1424 & 14.2586 \\
& 16F10.100DOF & 0.0000 & 0.0191 & 0.0071 & 0.0462 & 0.8402 & 6.0337 \\
& 4F16.52DOF & 0.0000 & 0.0328 & 0.3451 & 0.8392 & 4.0030 & 7.8597 \\
& 8F16.100DOF & 0.0000 & 0.0022 & 0.0339 & 0.3641 & 1.4248 & 2.5285 \\
\hline
\end{tabular}

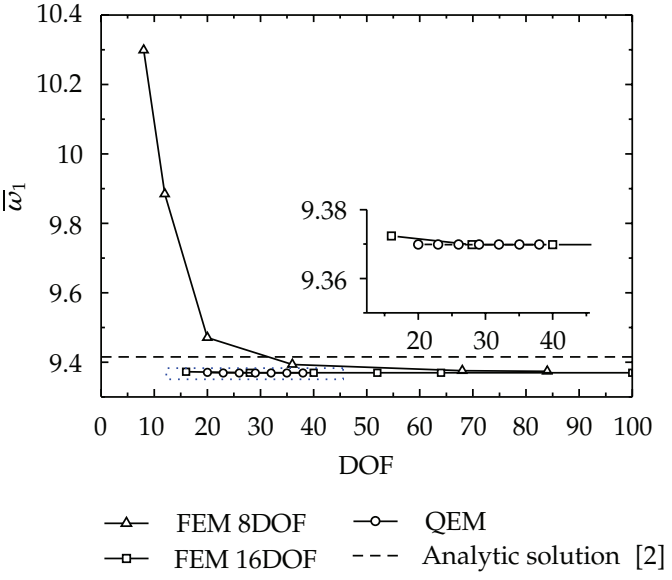

(a) $\alpha L=10$

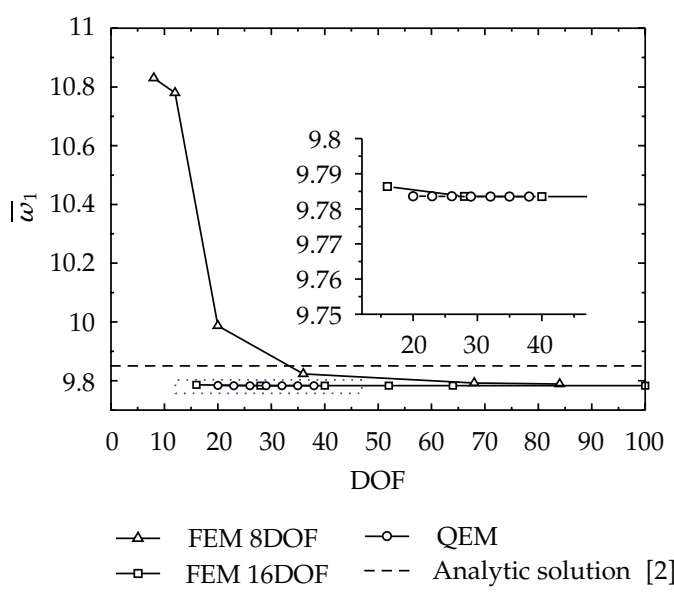

(b) $\alpha L=50$

Figure 5: Convergence of first order frequency of an SS beam.

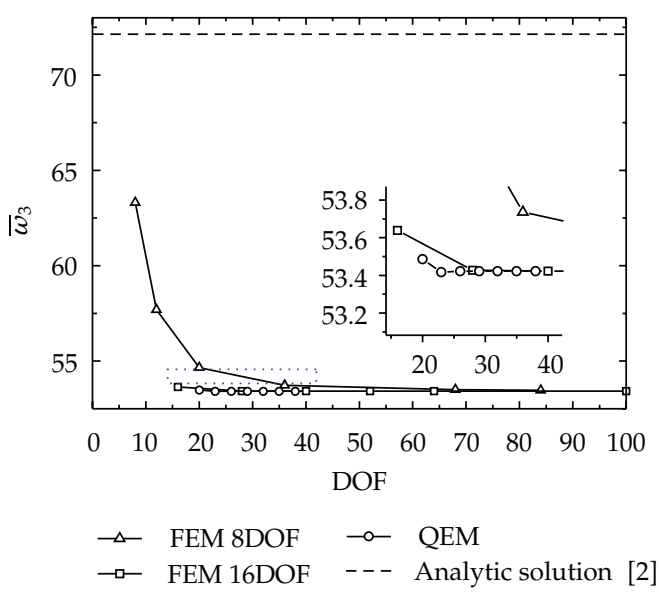

(a) $\alpha L=10$

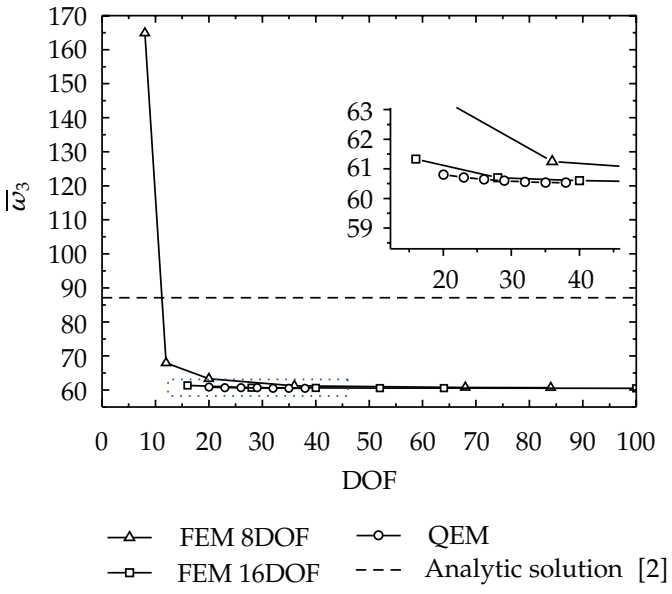

(b) $\alpha L=50$

Figure 6: Convergence of third order frequency of an SS beam. 


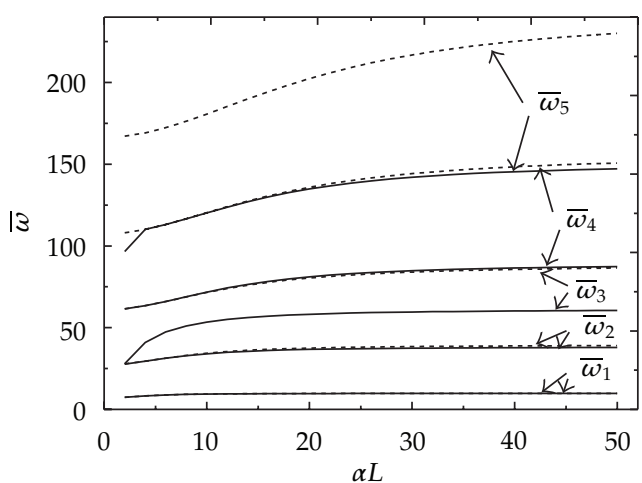

- With $T_{u}^{(e)}$

-- Without $T_{u}^{(e)}$

(a) SS

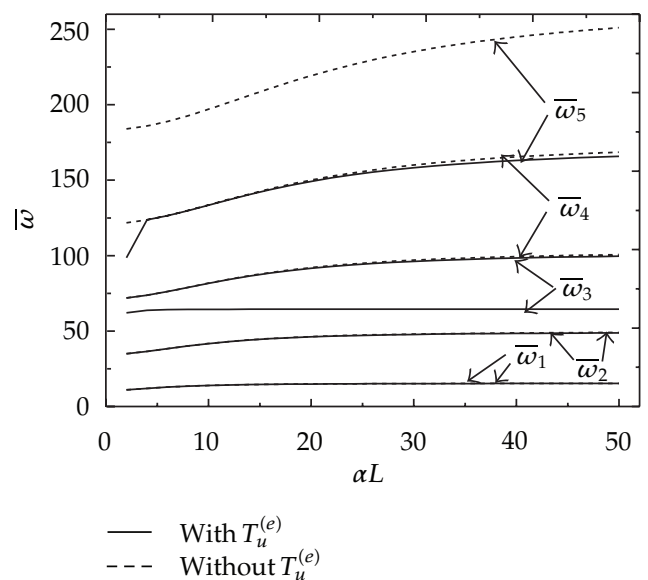

(c) $\mathrm{SC}$

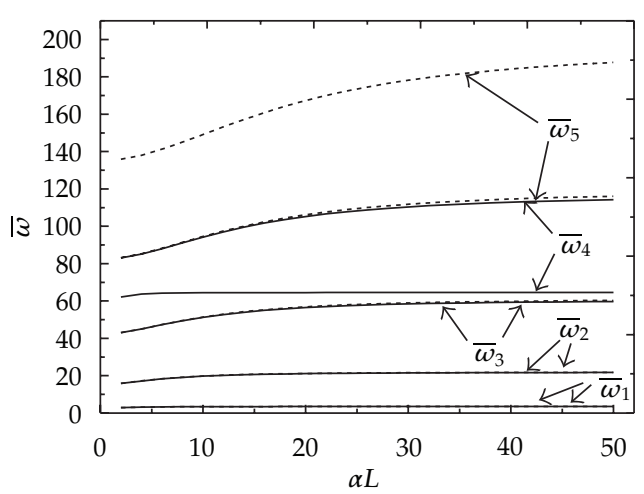

- With $T_{u}^{(e)}$

(b) FC

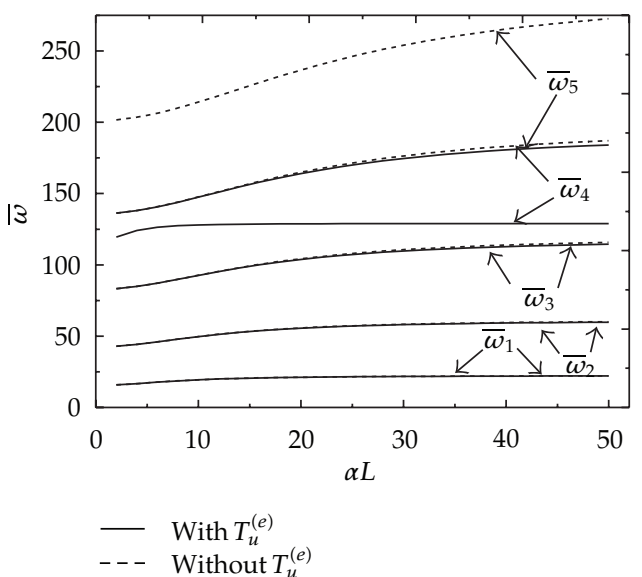

(d) $\mathrm{CC}$

Figure 7: First five natural frequencies of composite beam under four boundary conditions.

solutions and higher-order finite element formulations provide acceptable slip predictions for different shear connection levels. Nonetheless, the quadrature element solutions exhibit higher accuracy than those of finite element solutions given the same total number of degrees of freedom. It is found that low-order finite element solutions suffer oscillations especially for beams with large shear connection stiffness which were also reported and explained in detail in [10].

To further validate the capacity of the QEM, a continuous two-span composite beam of 40 meters in length $(2 L)$ with the same geometric and material data as those of example one is investigated. The external loads are two concentrated load of $Q=500 \mathrm{kN}$ applied at the two midspans, respectively. The computed results of the maximum deflection and the maximum slip for various shear connection stiffnesses are listed and compared with finite element solutions in Tables 9 and 10. It is seen that similar observations as in example one are acquired and the high computational accuracy and efficiency of the QEM are verified. 


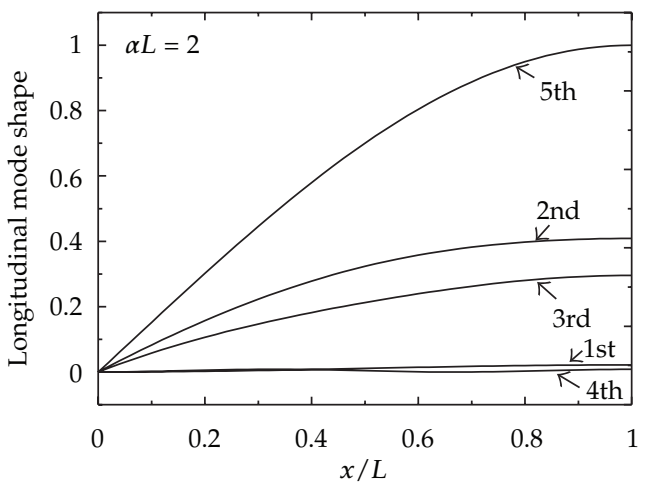

(a) Longitudinal mode shape $\alpha L=2$

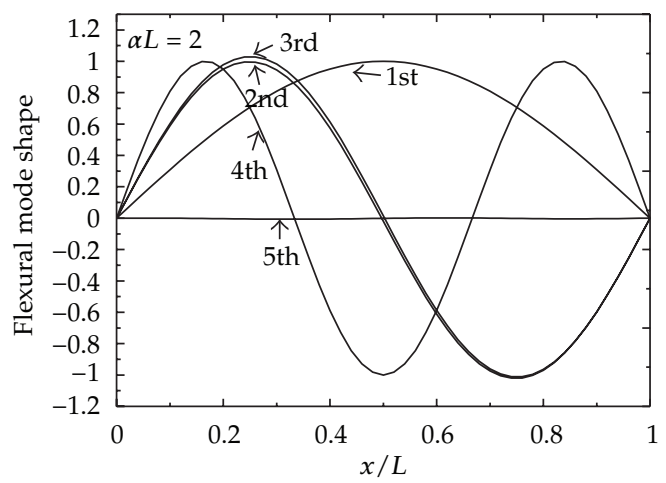

(c) Flexural mode shape $\alpha L=2$

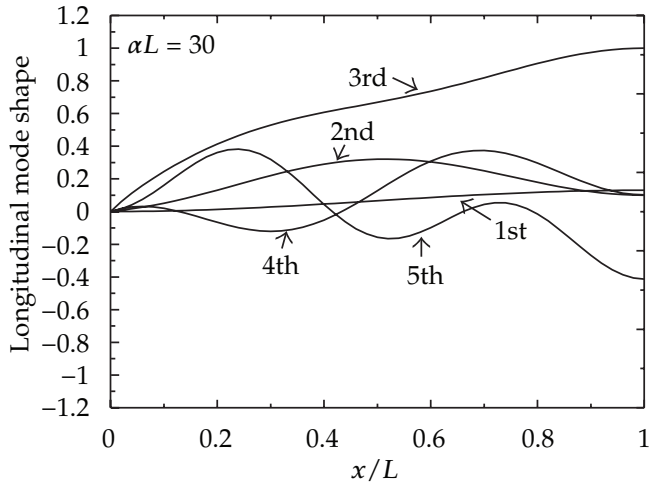

(b) Longitudinal mode shape $\alpha L=30$

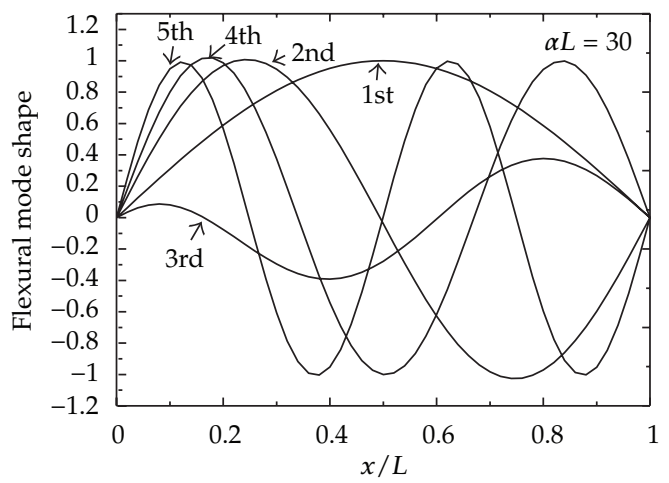

(d) Flexural mode shape $\alpha L=30$

Figure 8: First five mode shapes of SS composite beam for $\alpha L=2$ and $\alpha L=30$.

\subsection{Free Vibration Analysis}

For free vibration analysis of beams, it is assumed that

$$
\mathbf{d}=\mathbf{d}^{*} e^{i \omega t}
$$

Substitution of (3.4) and $\mathbf{F}=\mathbf{0}$ into (2.41) yields

$$
\left(\mathbf{K}-\omega^{2} \mathbf{M}\right) \mathbf{d}^{*}=\mathbf{0},
$$

which is a generalized eigenvalue problem. According to [24], a dimensionless frequency of the partially composite beam can be defined as

$$
\bar{\omega}=\omega L^{2}\left(\frac{\rho^{t} A^{t}+\rho^{b} A^{b}}{E I_{\infty}}\right)^{1 / 2}, \quad E I_{\infty}=E^{t} I^{t}+E^{b} I^{b}+\frac{E^{t} A^{t} E^{b} A^{b}}{E^{t} A^{t}+E^{b} A^{b}} h^{2} .
$$

For simplicity, a partially composite beam with the same geometric and material data as in Subsection 3.1 is adopted for free vibration analysis. First, an SS (both ends simply 


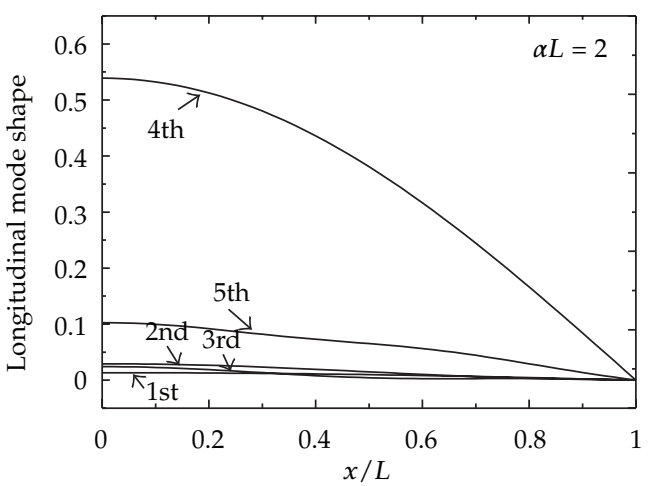

(a) Longitudinal mode shape $\alpha L=2$

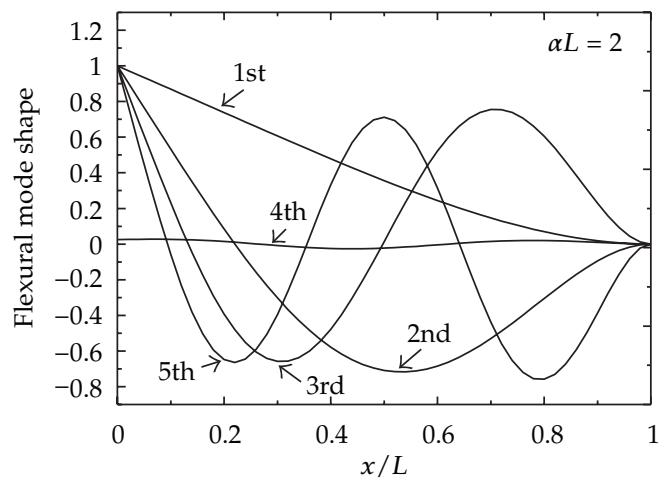

(c) Flexural mode shape $\alpha L=2$

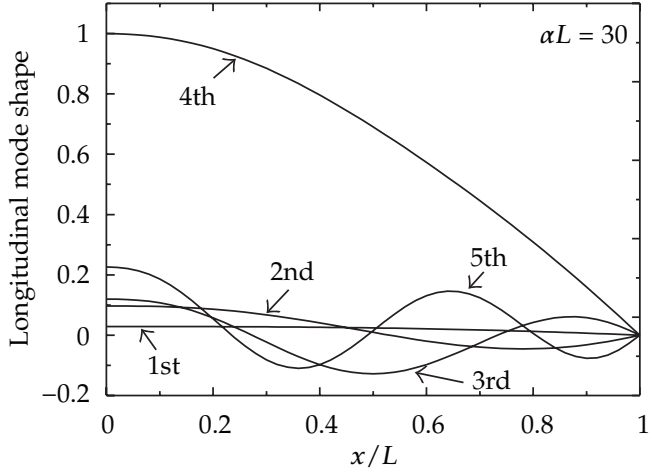

(b) Longitudinal mode shape $\alpha L=30$

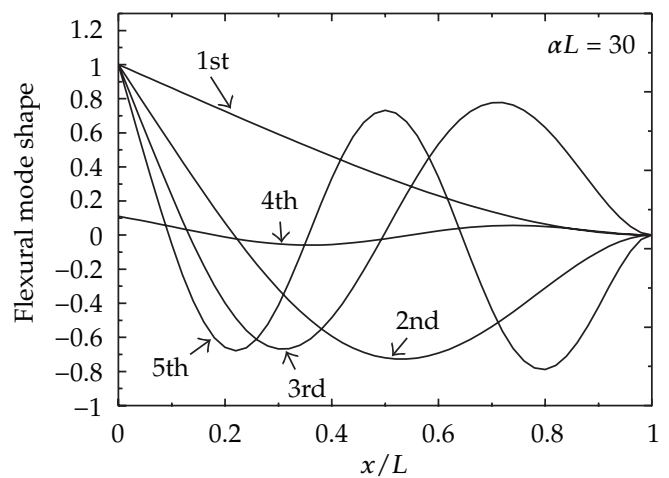

(d) Flexural mode shape $\alpha L=30$

Figure 9: First five mode shape of FC composite beam for $\alpha L=2$ and $\alpha L=30$.

supported) beam is considered to validate the convergence of the quadrature element solution. The first and the third dimensionless natural frequencies for two levels of connection stiffness are given in Figures 5 and 6.

It is seen that the quadrature element solution converges more rapidly than 8DOF and 16DOF finite element solutions and they agree with each other with the increase of total degrees of freedom. The 16DOF finite element can be used to acquire results with accuracy comparable with that of quadrature element solutions. However, a number of finite elements is needed in contrast to only one element in quadrature element analysis of partially composite beams. The dashed lines in Figures 5 and 6 are the analytical solutions given in [3] neglecting longitudinal inertias of motion $\left(T_{u}^{(e)}\right.$ in (2.31)). Obviously, omission of the longitudinal inertias of motion of the composite beam overestimates natural frequencies. Although the fundamental frequency of the beam is overestimated by less than $1 \%$, the thirdorder frequency is overestimated by $35 \%$ and $44 \%$ for small shear connection stiffness case $(\alpha L=10)$ and large shear connection stiffness case $(\alpha l=50)$, respectively.

To further investigate the dynamic behavior of partially composite beams, the effects of rotary inertia $\left(T_{r}^{(e)}\right.$ in $\left.(2.31)\right)$ and longitudinal inertia $\left(T_{u}^{(e)}\right.$ in $\left.(2.31)\right)$ on the frequencies and mode shapes of the beam are assessed. A quadrature element with 15 nodes (47 total degrees of freedom) is adopted to ensure the accuracy of solution. Although an allowance for rotary inertia results in reduction of frequencies of the beam, the amount of reduction is 


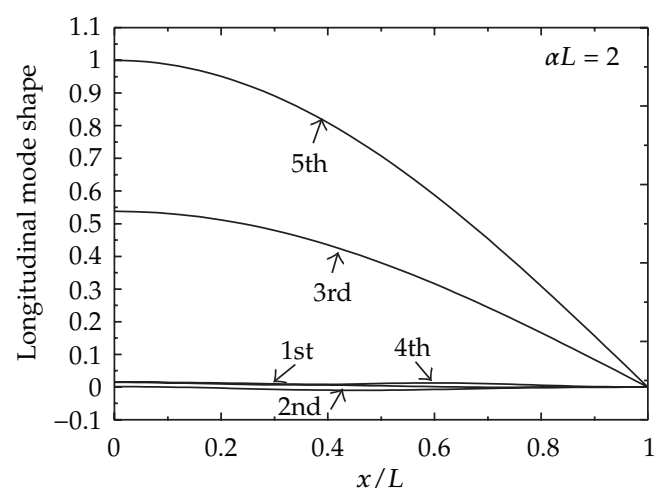

(a) Longitudinal mode shape $\alpha L=2$

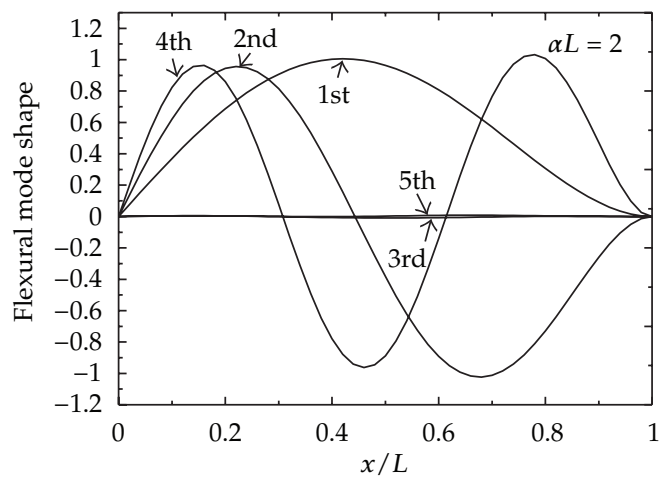

(c) Flexural mode shape $\alpha L=2$

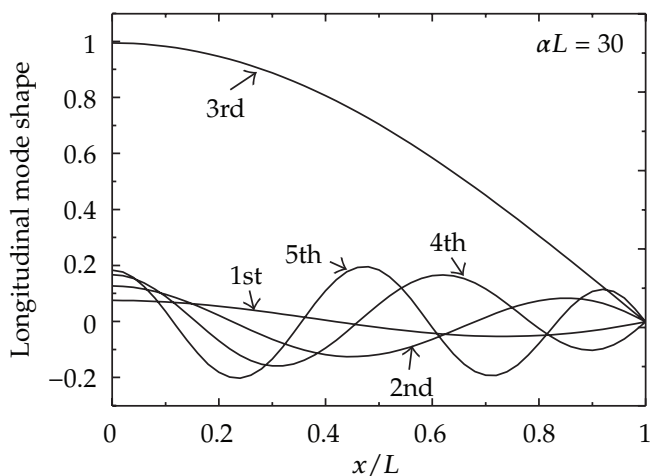

(b) Longitudinal mode shape $\alpha L=30$

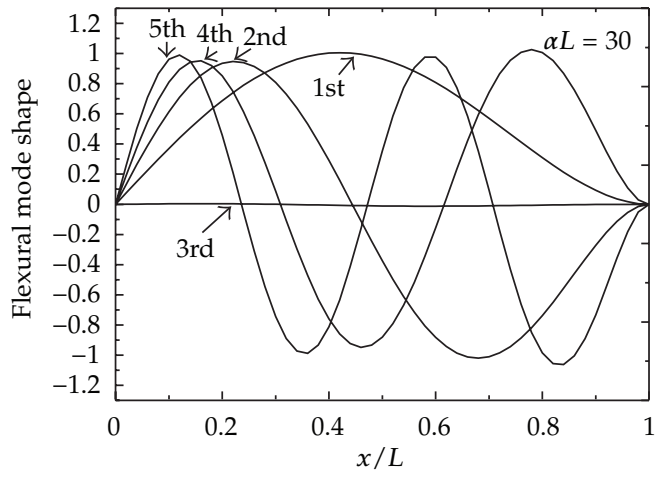

(d) Flexural mode shape $\alpha L=30$

Figure 10: First five mode shape of SC composite beam for $\alpha L=2$ and $\alpha L=30$.

small especially for slender beams like the one in question. It is observed that the reduction of the fifth frequency of the simply supported composite beam, the largest of the first five frequencies, is less than $1.5 \%$, indicating the negligible effect of the rotary inertia for loworder frequencies of the beam. The longitudinal inertia, on the other hand, has to be included in dynamic analysis since it can lead to significant change of vibration mode shapes as well as frequency characteristics of the beam.

As shown in Figure 7, ignoring longitudinal inertia leads to overestimation of frequencies; the higher the frequency order, the larger frequency overestimation which increases with shear connection stiffness and gradually levels off. It is noted that the first two frequencies for SS and SC beams and the first three frequencies for FC and CC beams are virtually not affected by the longitudinal inertia of the beam.

In Figures 8, 9, 10, and 11, the first five longitudinal and flexural modes of the beam under four different boundary conditions are displayed for small shear connection stiffness $\alpha L=2$ and large shear connection stiffness $\alpha L=30$, respectively. It is seen that in most cases longitudinal and flexural modes are coupled despite occasional predominance of either longitudinal or flexural mode for a certain-order frequency of partially composite beams. In addition, the longitudinal mode and the flexural mode for a certain-order frequency are more likely coupled with the increase of the shear connection stiffness or beam length. Since the longitudinal mode may appear at low-order frequencies of the composite beam (see 


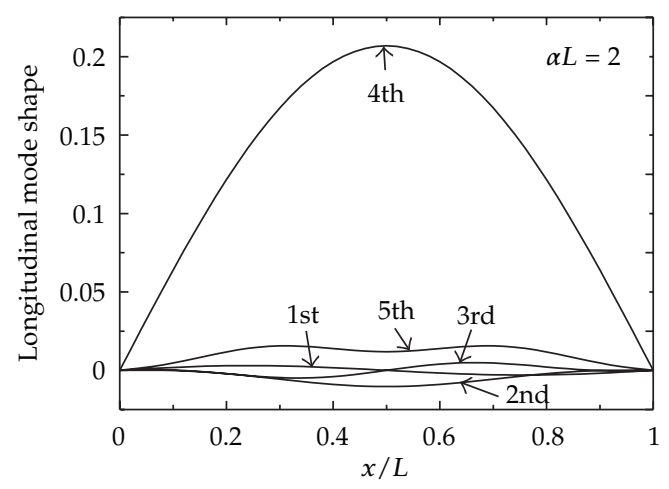

(a) Longitudinal mode shape $\alpha L=2$

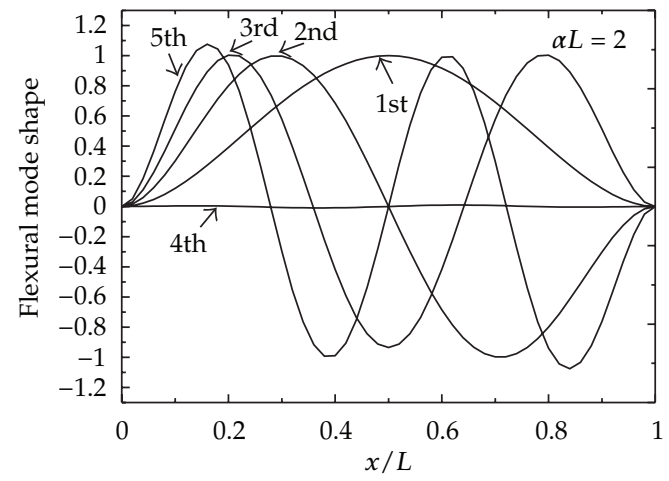

(c) Flexural mode shape $\alpha L=2$

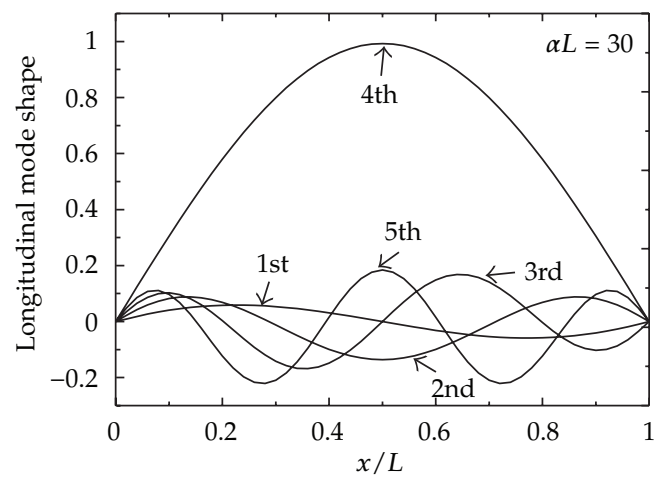

(b) Longitudinal mode shape $\alpha L=30$

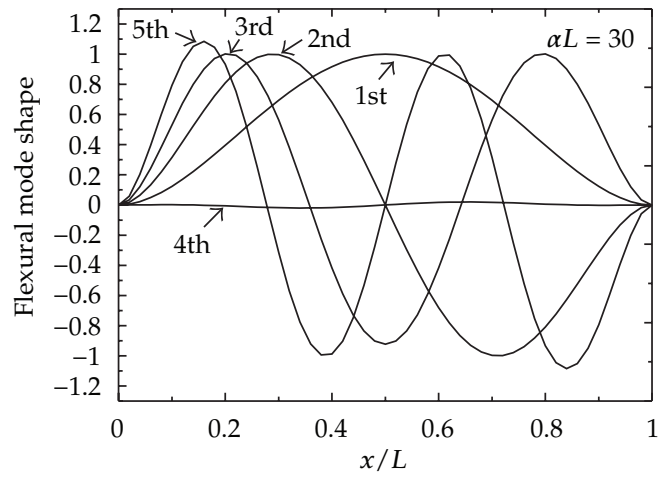

(d) Flexural mode shape $\alpha L=30$

Figure 11: First five mode shapes of CC composite beam for $\alpha L=2$ and $\alpha L=30$.

Figure 10), it is concluded that longitudinal inertia of motion cannot be simply neglected in dynamic analysis of partially composite beams. The effect of cross-sectional dimensions of the joist on the first five frequencies and mode shapes are shown in Figure 12. It can be seen that longitudinal modes may appear in low-order frequencies when the flexural rigidity of the joist is relatively large.

\section{Concluding Remarks}

Static and free vibrational analyses of partially composite beams have been studied using the weak-form quadrature element method. It has been shown that the weak-form quadrature element method works very well for partially composite beams with any level of connection stiffness. One quadrature element is usually sufficient for a partially composite beam under distributed load and the number of nodes is adjustable in accordance with the convergence requirement. However, division is clearly needed for quadrature element analysis of a multispan beam as well as a single-span beam under complex loadings like stepwise distributed loads. It is found that longitudinal inertia of motion cannot be simply neglected in dynamic analysis of partially composite beams since low-order frequencies as well as the mode shapes may be significantly affected. Although the QEM applications presented in this paper are limited to static and free vibration analysis, the extension of the present work to further 


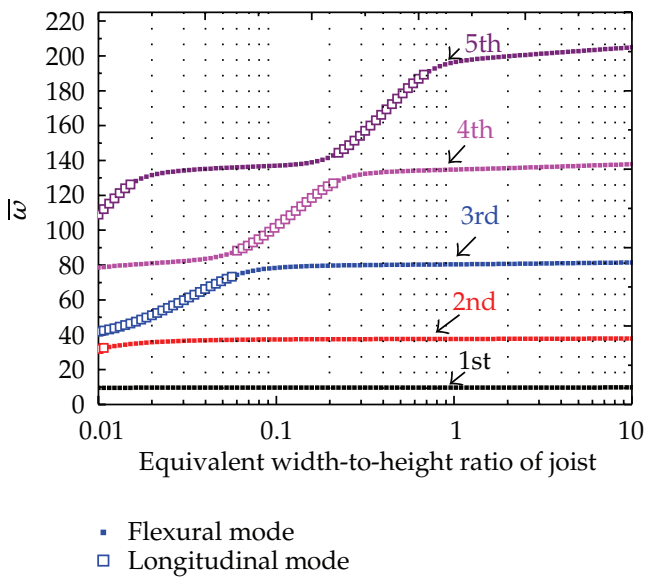

(a) Longitudinal mode shape $\alpha L=2$

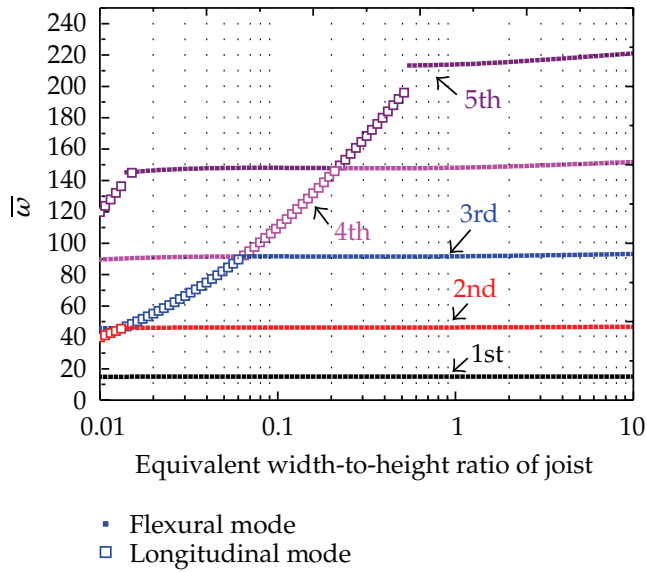

(c) Flexural mode shape $\alpha L=2$

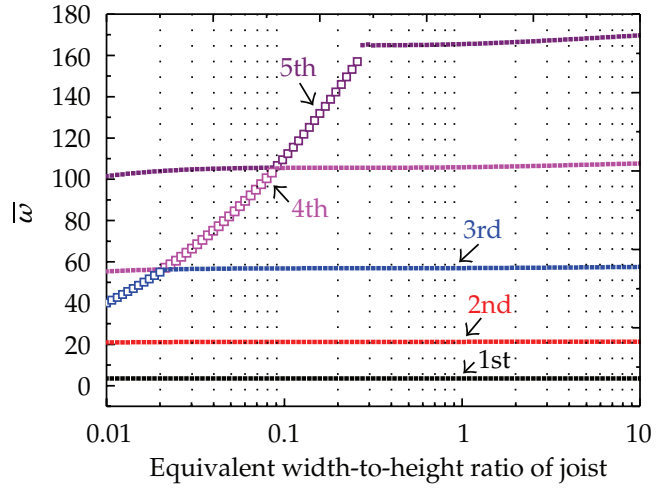

- Flexural mode

$\square$ Longitudinal mode

(b) Longitudinal mode shape $\alpha L=30$

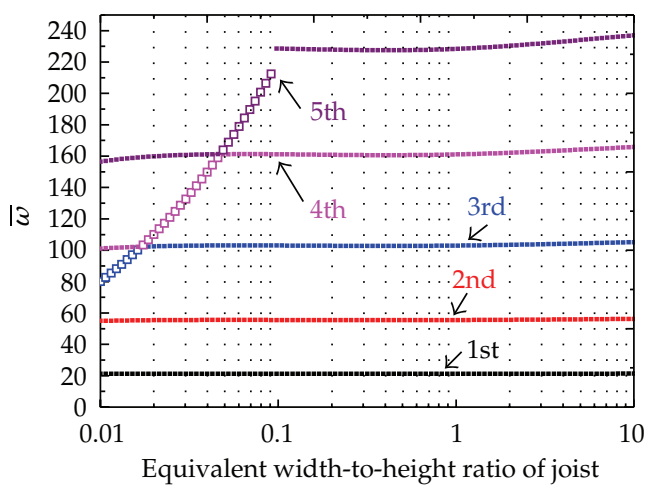

- Flexural mode

$\square$ Longitudinal mode

(d) Flexural mode shape $\alpha L=30$

Figure 12: Variation of frequencies and mode shapes versus aspect ratio of joist.

dynamic analysis, geometric, and material nonlinear analysis of partially composite beams is straightforward and will be reported in an upcoming paper.

\section{Acknowledgment}

This study was undertaken under the support of the National Natural Science Foundation of China (no. 51178247).

\section{References}

[1] N. M. Newmark, C. D. Siess, and I. M. Viest, "Tests and analysis of composite beams with incomplete interaction," Proceedings of the Society for Experimental Stress Analysis, vol. 9, pp. 75-92, 1951.

[2] U. A. Girhammar and D. H. Pan, "Exact static analysis of partially composite beams and beamcolumns," International Journal of Mechanical Sciences, vol. 49, no. 2, pp. 239-255, 2007.

[3] U. A. Girhammar, D. H. Pan, and A. Gustafsson, "Exact dynamic analysis of composite beams with partial interaction," International Journal of Mechanical Sciences, vol. 51, no. 8, pp. 565-582, 2009. 
[4] R. Xu and Y. Wu, "Static, dynamic, and buckling analysis of partial interaction composite members using Timoshenko's beam theory," International Journal of Mechanical Sciences, vol. 49, no. 10, pp. 11391155, 2007.

[5] A. Kroflič, I. Planinc, M. Saje, G. Turk, and B. Čas, "Non-linear analysis of two-layer timber beams considering interlayer slip and uplift," Engineering Structures, vol. 32, no. 6, pp. 1617-1630, 2010.

[6] J. M. Battini, Q. H. Nguyen, and M. Hjiaj, "Non-linear finite element analysis of composite beams with interlayer slips," Computers and Structures, vol. 87, no. 13-14, pp. 904-912, 2009.

[7] J. B. M. Sousa, C. E. M. Oliveira, and A. R. da Silva, “Displacement-based nonlinear finite element analysis of composite beam-columns with partial interaction," Journal of Constructional Steel Research, vol. 66, no. 6, pp. 772-779, 2010.

[8] G. Ranzi, "Locking problems in the partial interaction analysis of multi-layered composite beams," Engineering Structures, vol. 30, no. 10, pp. 2900-2911, 2008.

[9] G. Ranzi, F. Gara, G. Leoni, and M. A. Bradford, "Analysis of composite beams with partial shear interaction using available modelling techniques: a comparative study," Computers and Structures, vol. 84, no. 13-14, pp. 930-941, 2006.

[10] A. Dall'Asta and A. Zona, "Slip locking in finite elements for composite beams with deformable shear connection," Finite Elements in Analysis and Design, vol. 40, no. 13-14, pp. 1907-1930, 2004.

[11] H. Zhong and T. Yu, "Flexural vibration analysis of an eccentric annular Mindlin plate," Archive of Applied Mechanics, vol. 77, no. 4, pp. 185-195, 2007.

[12] H. Zhong and T. Yu, "A weak form quadrature element method for plane elasticity problems," Applied Mathematical Modelling, vol. 33, no. 10, pp. 3801-3814, 2009.

[13] H. Zhong and Y. Wang, "Weak form quadrature element analysis of Bickford beams," European Journal of Mechanics/Solids, vol. 29, no. 5, pp. 851-858, 2010.

[14] H. Zhong and M. Gao, "Quadrature element analysis of planar frameworks," Archive of Applied Mechanics, vol. 80, no. 12, pp. 1391-1405, 2010.

[15] T. Rabczuk, G. Zi, S. Bordas, and H. Nguyen-Xuan, “A geometrically non-linear three-dimensional cohesive crack method for reinforced concrete structures," Engineering Fracture Mechanics, vol. 75, no. 16, pp. 4740-4758, 2008.

[16] T. Rabczuk and T. Belytschko, "Application of particle methods to static fracture of reinforced concrete structures," International Journal of Fracture, vol. 137, no. 1-4, pp. 19-49, 2006.

[17] T. Rabczuk, J. Akkermann, and J. Eibl, "A numerical model for reinforced concrete structures," International Journal of Solids and Structures, vol. 42, no. 5-6, pp. 1327-1354, 2005.

[18] T. Rabczuk and T. Belytschko, "A three-dimensional large deformation meshfree method for arbitrary evolving cracks," Computer Methods in Applied Mechanics and Engineering, vol. 196, no. 29-30, pp. 27772799, 2007.

[19] P. J. Davis and P. Rabinowitz, Methods of Numerical Integration, Computer Science and Applied Mathematics, Academic Press, Orlando, Fla, USA, 2nd edition, 1984.

[20] R. Bellman and J. Casti, "Differential quadrature and long-term integration," Journal of Mathematical Analysis and Applications, vol. 34, pp. 235-238, 1971.

[21] J. R. Quan and C. T. Chang, "New insights in solving distributed system equations by the quadrature method-I. Analysis," Computers and Chemical Engineering, vol. 13, no. 7, pp. 779-788, 1989.

[22] C. W. Bert and M. Malik, "Differential quadrature method in computational mechanics: a review," Applied Mechanics Reviews, vol. 49, no. 1, pp. 1-27, 1996.

[23] C. Shu, Differential Quadrature and Its Application in Engineering, Springer, London, UK, 2000.

[24] Y. F. Wu, R. Xu, and W. Chen, "Free vibrations of the partial-interaction composite members with axial force," Journal of Sound and Vibration, vol. 299, no. 4-5, pp. 1074-1093, 2007. 


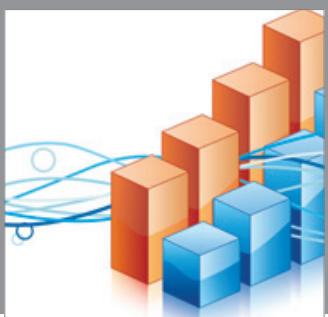

Advances in

Operations Research

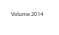

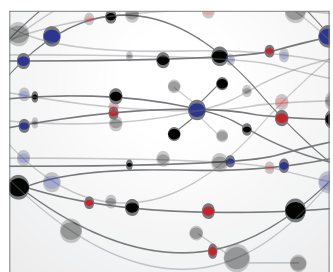

\section{The Scientific} World Journal
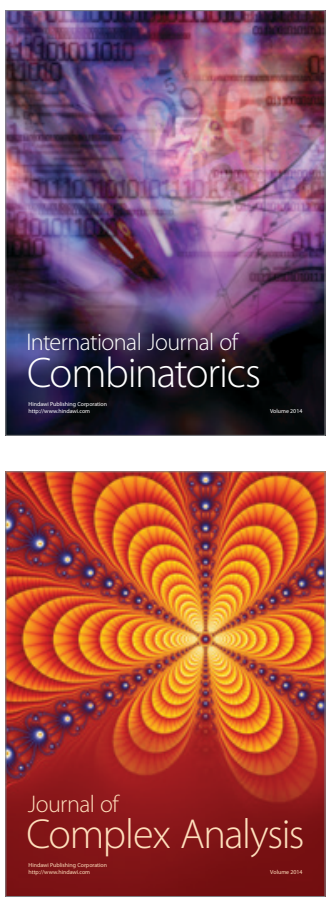

International Journal of

Mathematics and

Mathematical

Sciences
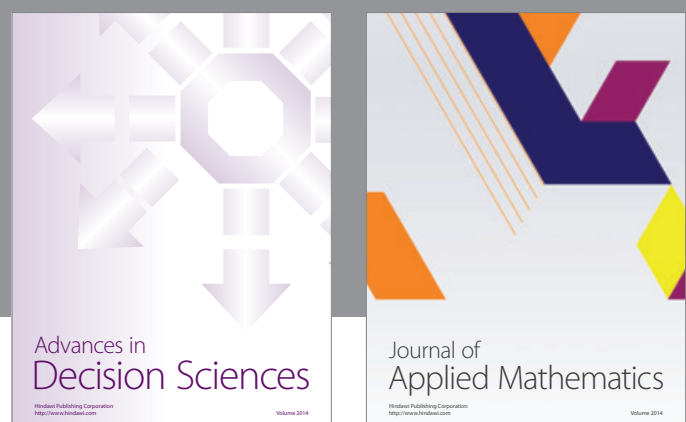

Journal of

Applied Mathematics
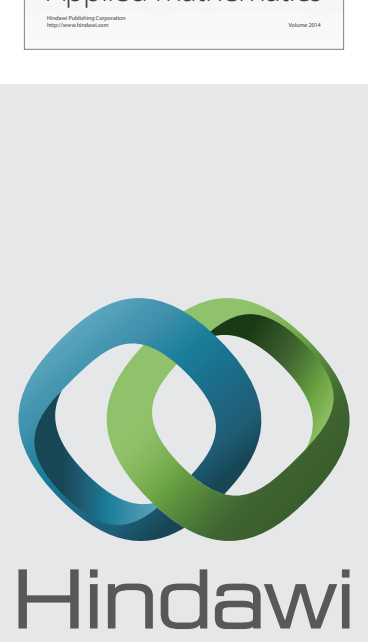

Submit your manuscripts at http://www.hindawi.com
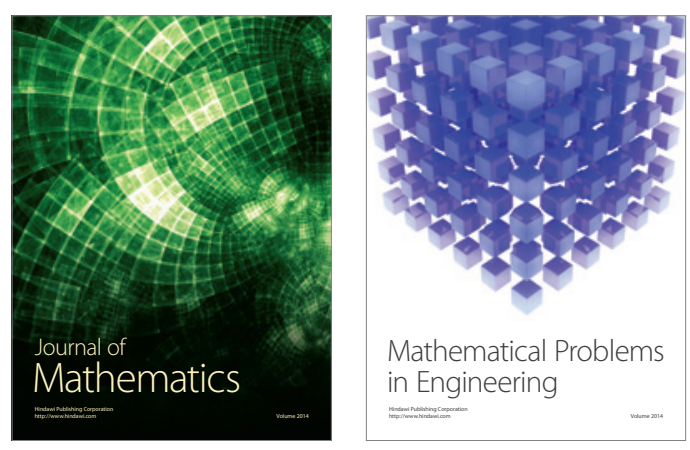

Mathematical Problems in Engineering
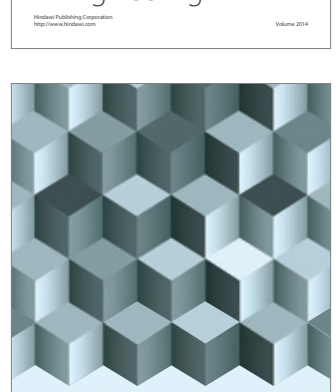

Journal of

Function Spaces
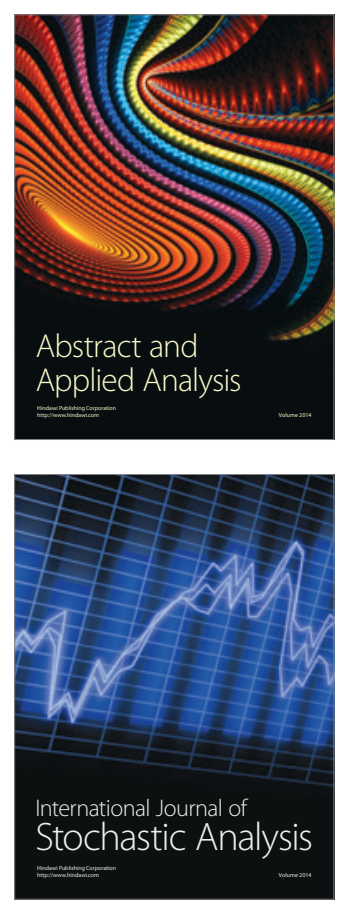

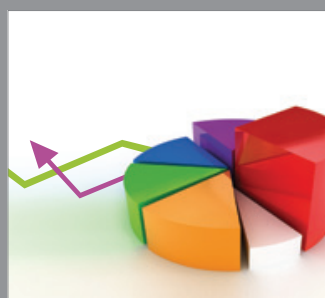

ournal of

Probability and Statistics

Promensencen
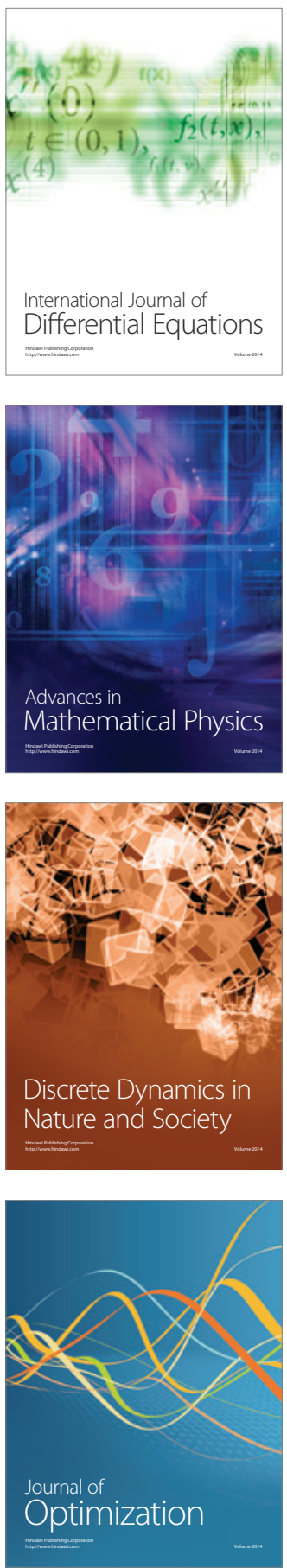